Was ist Globalisierung? Wie lässt sich das Verhältnis dieses Bündels weltumspannender Basisprozesse und der Politik, sie seit den 1990er Jahren zu beeinflussen und zu steuern, angemessen beschreiben? Jan Eckel richtet im dritten Teil des VfZSchwerpunkts zum Thema Globalisierung das Augenmerk auf dieses kritische Spannungsverhältnis und leuchtet dabei den diskursiven Hintergrund der Sozial- und Außenpolitik der Regierungen von Bill Clinton in den USA, Tony Blair in Großbritannien und Gerhard Schröder in der Bundesrepublik aus. Der Tübinger Historiker kommt dabei zu einem überraschenden Ergebnis und attestiert dieser Politik Züge einer Self-Fulfilling Prophecy: Die Regierungen schufen die Bedingungen der Globalisierung, die sie angeblich zu einschneidenden Reformen zwangen, durch ihr Handeln teilweise erst oder verstärkten sie zumindest. Diese Deutung wirft ein kritisches Schlaglicht auf angebliche Kausalitäten, die bei genauerem Hinsehen alles andere als klar auf der Hand liegen.

Jan Eckel

\title{
Politik der Globalisierung
}

Clinton, Blair, Schröder und die Neuerfindung der Welt in den 1990er und 2000er Jahren

\section{Die Geschichtlichkeit des Globalisierungsdenkens}

In einer Rede vom Januar 2000 umriss der amerikanische Präsident Bill Clinton die Kondition seiner Gegenwart:

„Heute, am Beginn eines neuen Jahrhunderts [...] steht die ganze Welt am Scheideweg. Die Globalisierung revolutioniert die Art, wie wir arbeiten, wie wir leben, und vielleicht am wichtigsten, die Art, wie wir über nationale Grenzen hinweg miteinander in Verbindung treten. Sie reißt Tore ein und schafft neue Vernetzungen zwischen Nationen und Individuen, zwischen Wirtschaften und Kulturen."1

Auch wenn das neue Jahrhundert in Wirklichkeit erst ein Jahr später beginnen sollte, war Clinton doch mit dem Begriff Globalisierung auf der Höhe der Zeit. In den zurückliegenden zehn Jahren war der Gedanke, die Regionen der Welt

\footnotetext{
${ }^{1}$ Bill Clinton, Remarks to the World Economic Forum and a Question-and-Answer Session in Davos, Switzerland, 29.1.2000; www.presidency.ucsb.edu/documents/remarks-the-worldeconomic-forum-and-question-and-answer-session-davos-switzerland [4.2.2020]. Übersetzung des Zitats von Jan Eckel. Wenn nicht anders gekennzeichnet, dann stammen Übersetzungen immer aus seiner Feder.
} 
wüchsen in atemberaubendem Tempo zusammen, zu einer Leitvorstellung westlicher - und anderer - Gesellschaften geworden und hatte sich im Terminus Globalisierung kristallisiert. Zuvor war der Begriff seit den 1940er Jahren vereinzelt im politischen und wissenschaftlichen Diskurs aufgetreten, ohne aber eine tragende Bedeutung zu erlangen. ${ }^{2}$ In der letzten Dekade des 20. Jahrhunderts dagegen begann er, die öffentliche Rede auf breiter Front zu durchdringen. In der Zeitdiagnostik und der medialen Berichterstattung avancierte er ebenso zu einem Angelpunkt der Beobachtung wie in der sozial- und geisteswissenschaftlichen Analyse und der kulturellen Reflexion. Gerade die besonders wichtigen Umbrüche und auffälligen Neuerungen, die sich in den Jahren um die Jahrtausendwende vollzogen, schienen mit der Globalisierung in Verbindung zu stehen: angefangen von den großen Weltproblemen wie Armut, Migration oder Erderwärmung über Krisenphänomene wie Börsencrashs und Währungsturbulenzen bis hin zu den Erscheinungen des alltäglichen Lebens - ob Fast-Food-Ketten oder Internet. $^{3}$

Die Globalisierungsrede diente indes nicht allein dazu, die Welt zu erklären, sondern auch, sie zu verändern. Im Lauf der 1990er und 2000er Jahre avancierten Fragen der Globalisierung zu einem prominenten Gegenstand der Politik. In westlichen Ländern bezogen Politikerinnen und Politiker jeder parteipolitischen Couleur Position, begründeten Maßnahmen damit und durchdachten die Folgen. In den USA, in Großbritannien und Deutschland gewann die politische Auseinandersetzung mit der offenbar zunehmenden weltweiten Integration einen besonders hohen Stellenwert. Die demokratische Regierung Bill Clintons (1993-2000), die Labour-Regierung unter Tony Blair (1997-2007) und die von Bundeskanzler Gerhard Schröder (Sozialdemokratische Partei Deutschlands, SPD) angeführte rotgrüne Koalition (1998-2005) formulierten umfassende sozial-, wirtschafts- und außenpolitische Reformagenden, die von dem Gedanken einer ausgreifenden Globalisierungsdynamik sowohl intellektuell als auch emblematisch zusammengehalten wurden - ganz so, wie es in Clintons Rede von Anfang 2000 aufschien. Somit trugen sie maßgeblich zu dem wachsenden Eindruck bei, die Hauptaufgabe einer zukunftsgerichteten Politik bestehe darin, Nationen und Gesellschaften so auszurichten, dass sie sich in einer globalisierten Welt behaupten könnten. Diesen Konnex luden sie emphatisch auf - was am stärksten für Tony Blair und New Labour galt - und riefen damit erbitterten innenpolitischen Streit hervor; das traf am meisten auf die Bundesrepublik zu. Beides sorgte dafür, dass die Frage nach dem rich-

\footnotetext{
${ }^{2}$ Vgl. Olaf Bach, Die Erfindung der Globalisierung. Entstehung und Wandel eines zeitgeschichtlichen Grundbegriffs, Frankfurt a. M./New York 2013. Dazu auch die beiden bereits erschienenen Texte des VfZ-Schwerpunkts: Olaf Bach, Ein Ende der Geschichte? Entstehung, Strukturveränderungen und die Temporalität der Globalisierungssemantik seit dem Zweiten Weltkrieg, in: VfZ 68 (2020), S. 128-154, und Wolfgang Knöbl, After Modernization. Der Globalisierungsbegriff als Platzhalter und Rettungsanker der Sozialwissenschaften, in: VfZ 68 (2020), S. 297-317.

${ }^{3}$ Vgl. Jan Eckel, ,Alles hängt mit allem zusammen.“ Zur Historisierung des Globalisierungsdiskurses der 1990er und 2000er Jahre, in: Historische Zeitschrift 307 (2018), S. 42-78.
} 
tigen Umgang mit der Globalisierung in den drei Ländern und darüber hinaus ins Zentrum der politischen Diskussion rückte.

Aus diesen Gründen bieten sich die drei Regierungen an, um dem Wechselverhältnis zwischen Globalisierungsdiskurs und Politik in den beiden Jahrzehnten um die Jahrhundertwende nachzugehen. Historische Studien über die Geschichte der USA, Großbritanniens und der Bundesrepublik in diesem Zeitraum haben die zeitgenössische Globalisierungsrede zumeist weitgehend übernommen. ${ }^{4}$ Sie sehen die drei Gesellschaften in der Regel auf dieselbe Art und Weise in grenzüberschreitenden Verflechtungsprozessen begriffen, wie es auch die Zeitgenossinnen und Zeitgenossen verstanden, bringen dafür dieselben Ursachen in Anschlag und erkennen dieselben Folgen, die bereits damals festgestellt wurden. Der aktuelle Schub an Studien zur jüngsten amerikanischen Zeitgeschichte weicht von diesem Muster insofern ab, als der Globalisierungsbegriff keine tragende Bedeutung gewinnt. Stattdessen erkennen sie die zentrale politisch-gesellschaftliche Dynamik der letzten Jahrzehnte in einem fortgesetzten Prozess der Polarisierung. Diesen machen sie an der unversöhnlichen Konfrontation zwischen den beiden großen parteipolitischen Lagern fest, an der wachsenden ökonomischen Ungleichheit, den immer heftigeren Culture Wars etwa um Sexualität und Gendernormen sowie an den zunehmend von einer radikalisierten Rechten gewaltsam geführten Konflikten um die gesellschaftliche Stellung der Afroamerikaner und anderer ethnischer Minderheiten. ${ }^{5}$ In jedem Fall ist in der jüngsten Forschung somit außer Betracht geblieben, dass die Rede von der Globalisierung ihrerseits ein historisches Produkt darstellt, das es zu erklären und zu erschließen gilt, ganz so wie andere ähnlich einflussreiche Diskurse auch - ob es sich um das Fortschrittsbewusstsein des 19. Jahrhunderts, das Ordnungsdenken der Zwischenkriegszeit, die Modernisierungseuphorie der 1960er Jahre, die periodisch wiederkehrenden Krisendiskurse oder die Vorstellung vom Ende der Geschichte handelt. Sie alle dienten dazu, den Wandel der Zeit zu deuten, einzuordnen - und eben auch zu beeinflussen.

\footnotetext{
${ }^{4}$ Vgl. Andreas Wirsching, Der Preis der Freiheit. Geschichte Europas in unserer Zeit, München 2012; ders., Demokratie und Globalisierung. Europa seit 1989, München 2015; Edgar Wolfrum, Rot-Grün an der Macht. Deutschland 1998-2005, München 2013; Ulrich Herbert, Geschichte Deutschlands im 20. Jahrhundert, München 2014; Alfred E. Eckes, Jr./Thomas W. Zeiler, Globalization and the American Century, Cambridge 2003; John Dumbrell, Clinton's Foreign Policy. Between the Bushes, 1992-2000, London/New York 2009; Adam Tooze, Crashed. Wie zehn Jahre Finanzkrise die Welt verändert haben, München 2018; Ian Kershaw, Achterbahn. Europa 1950 bis heute, München 2019, und Simon Reid-Henry, Empire of Democracy. The Remaking of the West since the Cold War, 1971-2018, New York 2019.

${ }^{5}$ Vgl. Jill Lepore, These Truths. A History of the United States, New York 2018, S. 521-784, und Kevin M. Kruse/Julian E. Zelizer, Fault Lines. A History of the United States Since 1974, New York 2019. Dass mit der Polarisierung ebenfalls eine zeitgenössische Interpretationsfigur aufgenommen wird, betonte Thomas Zimmer, Reflections on the Challenges of Writing a (Pre-) History of the „Polarized“ Present, in: Modern American History 2 (2019), S. 403-408. Vgl. auch Glenda Elizabeth Gilmore/Thomas J. Sugrue, These United States. A Nation in the Making, 1890 to the Present, New York 2015, S. 623-625.
} 
So verstanden, bildet der Globalisierungsdiskurs einen wichtigen analytischen Schlüssel zum historischen Verständnis der 1990er und 2000er Jahre. Er führt ins Zentrum der Vorstellungen und Debatten darüber, wie sich die Veränderungsdynamik der Jahrtausendwende gesellschaftlich auswirke und wie sie sich politisch steuern lasse. Aus dieser Perspektive geht es nicht um die Geschichte der Globalisierung in dem Sinn, weiträumige Verknüpfungen und deren Konsequenzen für das Leben und Handeln nachzuvollziehen. In den Mittelpunkt rücken vielmehr die Fragen, wie sich die Ideen über die Globalisierung entwickelten, welche Auffassungen des Wandels sich dahinter verbargen, wie diese die politische Gegenwartsdeutung prägten und wie sie sich in politischen Entscheidungen niederschlugen.

Um die politische Wirkmacht der Globalisierungsvorstellung zu bestimmen, gilt es in einem ersten Schritt zu untersuchen, wie die Diagnose einer globalisierten Welt in der amerikanischen, britischen und deutschen Regierung aufkam und welche konzeptionelle Bedeutung sie gewann. Anschließend soll anhand zweier Politikfelder (und einem Seitenblick auf die amerikanische Freihandelspolitik) beleuchtet werden, auf welche Weise das Globalisierungsdenken die politische Praxis formte. Der Komplex der Sozialstaats- und Arbeitsmarktreformen gehörte in allen drei Ländern zu den wichtigsten Regierungsvorhaben. Er löste anhaltende, von breitgefächertem Expertenwissen gestützte politische Diskussionen aus und war nicht zuletzt in der Öffentlichkeit heftig umstritten. Daher steht er im Zentrum der Untersuchung. Wenngleich zeitgenössisch weniger kommentiert, beeinflussten Globalisierungsdiagnosen jedoch auch die außenpolitischen Neupositionierungen, die die Regierungen in der veränderten weltpolitischen Situation nach dem Ende des Kalten Kriegs für nötig hielten. Sie sollen daher ebenfalls, wenn auch knapper, beleuchtet werden. Damit rücken Bereiche der amerikanischen, britischen und deutschen Politik in den Blick, in denen sich während der 1990er und frühen 2000er Jahre besonders weitreichende Umbrüche ereigneten.

\section{Auf der Suche nach neuen Kategorien}

Am Anfang und in der Mitte der 1990er Jahre befanden sich die Demokratische Partei in den USA, die Sozialisten in Großbritannien und die deutsche Sozialdemokratie in einer strukturell ähnlichen Situation. Alle sahen sich mit einem doppelten Erneuerungsbedarf konfrontiert. Zunächst einmal mussten sich die Parteien angesichts langer oppositioneller Durststrecken neu erfinden - die Demokraten wollten nach zwölf, Labour nach 18 und die SPD nach 16 Jahren die Regierungsmacht zurückgewinnen. Das löste ein intensives Bemühen um programmatische Neudefinition aus, die mit einem deutlichen Zug in die politische Mitte verbunden war. Ein wichtiger Schritt in diese Richtung bestand darin, dass die Parteien ihre wirtschaftliche Kompetenz akzentuierten. Bill Clinton legte sich früh darauf fest, das Haushaltsdefizit zu reduzieren, und grenzte sich damit unverkennbar von den (abschätzig so bezeichneten) Tax and Spend Liberals ab, den vermeintlich ausgabefreudigen linksliberalen Befürwortern einer ausgedehnten Sozialpolitik, mit 
denen er nicht identifiziert werden wollte. ${ }^{6}$ Die Labour-Partei, deren Re-Orientierung bereits nach der Wahlniederlage von 1992 begonnen hatte, bevor sie Tony Blair als neuer Vorsitzender ab 1994 entschlossen vorantrieb, bekannte sich zu einer zurückhaltenden Ausgabenpolitik und erteilte Steuererhöhungen eine Absage. ${ }^{7}$ Diese ökonomische Positionierung ging mit einer positiven Aneignung von Teilen der Politik einher, welche die konservativen Vorgängerregierungen betrieben hatten - diese „hatten einiges richtig gemacht“, wie Tony Blair mit Blick auf die ehemalige Premierministerin Margaret Thatcher formulierte. ${ }^{8}$ Einen neuralgischen Punkt berührte der demokratische Präsident Clinton, als er das Ziel ausgab, „den Wohlfahrtsstaat, wie wir ihn kennen, abzuschaffen“. ${ }^{9}$ Damit brachte er einen Plan früh und offensiv auf eine Formel, den die Labour-Regierung und die rotgrüne Koalition später ebenfalls umsetzen sollten. Auf diese Weise brachen alle Parteien gleich mehrfach bewusst mit jahrzehntealten Traditionen, indem sie ihr politisches Selbstbild umkrempelten, von eingeschliffenen Konfliktmustern abwichen und sich de facto von Teilen ihrer alten politischen Klientel distanzierten. Blair spitzte diese politische Neuausrichtung noch zu, indem er eine ostentativ anti-ideologische Haltung einnahm. Den Rechts-Links-Gegensatz erklärte er für historisch überwunden und postulierte: „Die Ära der großen Ideologien - allumfassend, alles durchdringend, total in ihren Lösungen und oft gefährlich - ist vorbei. ${ }^{10}$ Das neue Projekt wurde bald auf eine griffige Formel gebracht: der Dritte Weg. ${ }^{11}$

In dieser Bestimmung deutet sich bereits an, dass über die Notwendigkeit der parteipolitischen Profilierung hinaus auch der Verlust an fester weltanschaulicher Orientierung noch nachwirkte, der mit dem Ende des Systemgegensatzes am Anfang der 1990er Jahre zusammenhing. Mit dem Verschwinden des osteuropäischen Kommunismus hatte sich auch ein politisch-intellektueller Denkrahmen aufgelöst, der die Beobachtung der nationalen und internationalen Ordnung über Jahrzehnte tief geprägt hatte. In den außenpolitischen Überlegungen der amerikanischen Regierung wurde dies am unmittelbarsten fassbar. Präsident Clinton war unzufrieden mit der verbreiteten Rede von der Post-Cold War Foreign Policy, welche die internationale Rolle der USA ausschließlich negativ beschrieb - mit Blick auf das, was sie nicht mehr war. Daher beauftragte er das State Department, eine Formel

\footnotetext{
6 Vgl. James T. Patterson, Restless Giant. The United States from Watergate to Bush v. Gore, Oxford 2005, S. 318-386, und Sean Wilentz, The Age of Reagan. A History, 1974-2008, New York 2008, S. 323-407.

7 Vgl. Anthony Seldon (Hrsg.), Blair's Britain. 1997-2007, Cambridge 2007, und Kenneth O. Morgan, The Twentieth Century (1914-2000)/Epilogue (2000-2010), in: Ders. (Hrsg.), The Oxford History of Britain, Oxford 2010, S. 582-710, hier S. 662-710.

8 Tony Blair, Security in a World of Change, 17.7.1995, in: Ders., New Britain. My Vision of a Young Country, London 1996, S. 203-214, hier S. 206.

9 Bill Clinton, Address Before a Joint Session of Congress, 17.2.1993; millercenter.org/thepresidency/presidential-speeches / february-17-1993-address-joint-session-congress [4.2.2020].

${ }^{10}$ Blair, Security in a World of Change, in: Ders., New Britain, S. 213.

${ }^{11}$ Vgl. Anthony Giddens, Der dritte Weg. Die Erneuerung der sozialen Demokratie, Frankfurt a. M. 1999.
} 
für die Essenz der neuen amerikanischen Außenpolitik zu finden, die ähnlich zugkräftig sei wie der Gedanke des Containment im Kalten Krieg. ${ }^{12}$

Ein grundlegendes Bedürfnis nach neuen Kategorien, welche die veränderten politischen Realitäten beschreiben und ordnen konnten, war somit im linken und linksliberalen Lager am Anfang der 1990er Jahre aus mehreren Gründen vorhanden. Was die weltanschaulich strukturierenden Denkfiguren ihrer Politik betraf, so operierten die Demokraten, Sozialisten und Sozialdemokraten anfänglich in einer Art Vakuum. Das stellte eine nicht zu unterschätzende Voraussetzung dafür dar, dass die Globalisierungsvorstellung so attraktiv erscheinen konnte. Tatsächlich bestimmte die Diagnose eines präzedenzlos schnellen und weltverändernden Wandels die Grundsatzbestimmungen in der neuen amerikanischen Regierung und bei New Labour schon einige Jahre, bevor sich dafür die Vokabel Globalisierung einbürgerte. Begrifflich fassten die Politiker dies zunächst als „globalen Wandel“, bezogen sich auf die Impulse der „globalen Wirtschaft" mit ihrem vermeintlich weltumspannenden Wettbewerb oder sprachen vom Entstehen einer "globalen Gesellschaft". ${ }^{13}$ Daraus gewannen sie ein weitreichendes Erneuerungsargument, das sie in den nächsten Jahren seiner Form nach unverändert beibehalten sollten. Ihm zufolge bestand die oberste Aufgabe der Politik darin, den jeden Maßstab sprengenden Veränderungsschüben der Gegenwart angemessen zu begegnen und die Weichen so zu stellen, dass sie sich zum Vorteil der eigenen Gesellschaft und der eigenen Nation entwickelten. „Tiefgreifende und machtvolle Kräfte schütteln unsere Welt durch und schaffen sie neu“, gab Bill Clinton in seiner Vereidigungsansprache von 1993 zu bedenken. „Und die drängende Frage unserer Zeit lautet, ob wir den Wandel zu unserem Freund machen können und nicht zu unserem Feind“. ${ }^{14}$ New Labour ließ es in seinem Wahlprogramm von 1997 geradezu als raison d'être der reformierten Partei erscheinen, „sich den Herausforderungen einer veränderten Welt zu stellen. [...] Dieses Großbritannien wird in der Lage sein, in einer von technologischem Wandel bestimmten globalen Wirtschaft zu prosperieren." 15

Der Begriff Globalisierung gelangte in allen drei Ländern 1995 in die politische Debatte. Tony Blair beschrieb sie vergleichsweise früh als eine nahezu allumfassende Ausgangsbedingung, mit der die Politik unabweislich zu rechnen habe: „Die Triebkraft des wirtschaftlichen Wandels ist heute die Globalisierung“, legte er im Juli desselben Jahrs dar:

${ }^{12}$ Vgl. James D. Boys, Clinton's Grand Strategy. U. S. Foreign Policy in a Post-Cold War World, London 2015.

${ }^{13}$ Vgl. Bill Clinton, Remarks on the House of Representatives Action on the North American Free Trade Agreement and an Exchange With Reporters, 17.11.1993; www.presidency.ucsb. edu/documents / remarks-the-house-representatives-action-the-north-american-free-tradeagreement-and [5.2.2020], und Blair, New Britain. Die Begriffe sind häufig in den beiden zitierten Texten vertreten, deshalb wird auf eine konkrete Seitenzahl verzichtet.

${ }^{14}$ Bill Clinton, Inaugural Address, 20.1.1993; www.presidency.ucsb.edu/documents /inauguraladdress-51 [5.2.2020].

${ }^{15}$ New Labour because Britain deserves better, 1997; www.labour-party.org.uk/manifestos/ 1997/1997-labour-manifesto.shtml [5.2.2020]. 


\begin{abstract}
„Technologie und Kapital sind mobil. Die Industrie steht über nationale Grenzen hinweg in einem scharfen Wettbewerb. [...] Reisen, Kommunikation und Kultur werden immer stärker international, wodurch die Welt schrumpft und sich Präferenzen, Wahlmöglichkeiten und Wissen ausdehnen. Die entscheidende Frage, der alle Regierungen der entwickelten Länder gegenüberstehen, ist, wie wir darauf antworten sollen.“16
\end{abstract}

Der gesamte Komplex bahnbrechender weltweiter Neuentwicklungen verfestigte sich alsbald im Topos der „Herausforderungen der Globalisierung“, der nun in den Mittelpunkt des Nachdenkens über notwendige politische Veränderungen zu rücken begann.

Dabei war es ein Kennzeichen der deutschen Entwicklung, dass sich zunächst eine Art Konkurrenz zwischen der konservativ-liberalen Regierung und der linken Opposition darum entspann, den Begriff zu besetzen. Seit 1996 bemühte die Regierung von Helmut Kohl (Christlich Demokratische Union Deutschlands, CDU) den Verweis auf die Globalisierung nachdrücklich, um ihre wirtschafts- und sozialpolitischen Reformkonzepte zu begründen, die von den sozialdemokratisch geführten Ländern im Bundesrat blockiert wurden. In den folgenden beiden Jahren gelang es jedoch der SPD und den Grünen, den Begriff für sich zu reklamieren und an ihre Reformagenda zu binden. Sie warfen der christlich-liberalen Regierung vor, „keine Antworten auf die Globalisierung“"17 zu besitzen und „tatenlos zuzusehen, wie die Globalisierung der Politik entgleitet“, ${ }^{18}$ während sie selbst über die zukunftsfähigen Rezepte verfügen würden. Damit wurde der Globalisierungsbegriff in der Bundesrepublik in den Jahren 1997/98 zu einem zentralen Schlagwort der politischen Kommunikation. ${ }^{19}$

Die frühen politischen Auseinandersetzungen kreisten um ein bestimmtes Syndrom weiträumiger Verflechtung, das fast stets als Wesenskern oder als vorrangige Triebkraft der Globalisierung angeführt wurde. Dabei handelte es sich um den noch näher zu erörternden Gestaltwandel der Weltwirtschaft, der wiederum an durchschlagende Neuerungen in den Bereichen Telekommunikation und Transporttechnologie gekoppelt wurde. ${ }^{20}$ In den späteren 1990er Jahren fächerte sich diese Bestandsaufnahme allerdings auf, so dass die Globalisierung bald als ein alle Lebensbereiche und Handlungsfelder durchwirkendes Prinzip erschien. Die Lo-

\footnotetext{
${ }^{16}$ Tony Blair, The Global Economy, 5.1.1996, in: Ders., New Britain, S. 118-129, hier S. 118.

${ }^{17}$ Deutscher Bundestag, Entschließungsantrag der SPD-Fraktion, Politische Antworten auf die wirtschaftliche Globalisierung, Bundestag-Drucksache 13/11011, 17.6.1998, S. 2; dipbt. bundestag.de/doc/btd/13/110/1311011.pdf [19.3.2020].

${ }^{18}$ Deutscher Bundestag, Große Anfrage der SPD-Fraktion, Politische Antworten auf die wirtschaftliche Globalisierung, Bundestag-Drucksache 13/10103, 6.3.1998, S. 2; dipbt.bundestag.de/dip21/btd/13/101/1310103.pdf [5.2.2020].

${ }^{19}$ Zu den USA vgl. Bill Clinton, The President's Radio Address, 3.1.1998; www.presidency.ucsb. edu/documents/the-presidents-radio-address-185 [5.2.2020].

${ }^{20}$ Vgl. ders., Remarks to the International Monetary Fund and the World Bank, 11.10.1995; www.presidency.ucsb.edu/documents / remarks-the-international-monetary-fund-and-theworld-bank [5.2.2020].
} 
gik der Globalisierung sei „nicht nur wirtschaftlich“, wie die Spitzenpolitiker nun unisono betonten. ${ }^{21}$ Vielmehr galt, in den Worten Clintons von Anfang 1999: „Alles von der Stärke unserer Wirtschaft über die Sicherheit unserer Städte bis zur Gesundheit unserer Menschen hängt von Dingen ab, die nicht nur innerhalb unserer Grenzen, sondern eine halbe Welt entfernt geschehen“. ${ }^{22}$ In dieser Sicht prägte das schleunige Zusammenwachsen der Welt etwa auch Mobilitätsformen, Arbeitsmuster, Familienmodelle, die Bedeutung von Religion, das Verhältnis zwischen globalem Norden und Süden, die neuen Gefährdungen der Umwelt oder die Bedrohung, die von „Schurkenstaaten“ ausging. Die Globalisierungsvorstellung war zu einer fast alle Politikfelder verbindenden Diagnose gereift. Dies kulminierte am Ende des Jahrhunderts schließlich darin, dass alle drei Regierungen eine „Ära der Globalisierung“ ausriefen. ${ }^{23}$ „Unsere politische Generation“, so verkündete Bundeskanzler Schröder in einer Regierungserklärung nach seiner Wiederwahl 2002, „steht vor der historischen Aufgabe, Gerechtigkeit im Zeitalter der Globalisierung zu definieren“. ${ }^{24}$

Die Globalisierungsrede blieb jedoch nicht dabei stehen, die Bewegungsprinzipien und den epochalen Charakter des gegenwärtigen Geschehens zu durchleuchten. Gleichzeitig erzeugte sie einen geradezu atemlosen Modus der politischen Problemwahrnehmung. Einen wichtigen Teil davon machte die Beschwörung vielfältiger Bedrohungen aus. Keine der Regierungen zeichnete ein ungetrübt positives Zukunftsbild. Die Globalisierung trug vielmehr, wie etwa der amerikanische Präsident betonte, „die Keime neuer Zerrüttung, neuer Instabilität, neuer Ungleichheiten“ in sich. ${ }^{25}$ Die Kehrseite des Prozesses in Form der Abwanderung von Jobs, von Finanzkrisen, der Umtriebe krimineller Netzwerke oder eskalierender Regionalkonflikte malten er und andere Regierungsvertreter eindringlich aus. Die größte Gefahr erkannten sie indes darin, sich dem Megaprozess zu verweigern, denn so würden ihre Gesellschaften irreversibel ins Hintertreffen geraten. Daher hing alles - Prosperität, Sicherheit, Lebensqualität - davon ab, die Globalisierung richtig zu gestalten.

Das erschien auch deshalb entscheidend zu sein, weil die Regierungen den Globalisierungsprozess als unausweichlich beschrieben. Sofern es um Sprachbilder ging, die das zum Ausdruck bringen sollten - von Naturphänomenen wie dem

${ }^{21}$ Vgl. ders., Address Before a Joint Session of the Congress on the State of the Union, 27.1.2000; www.presidency.ucsb.edu/documents/address-before-joint-session-the-congress-the-state-theunion-7 [5.2.2020].

${ }^{22}$ Ders., Remarks on United States Foreign Policy in San Francisco, 26.2.1999; www.presidency. ucsb.edu/documents/remarks-united-states-foreign-policy-san-francisco [5.2.2020].

${ }^{23}$ Speech by the Prime Minister, the Right Honorable Tony Blair MP „Values and the Power of Community" to the Global Ethics Foundation Tübingen University, 30.6.2000; www.weltethos. org/1-pdf/20-aktivitaeten/eng/we-reden-eng/speech_Blair_eng.pdf [5.2.2020].

${ }^{24}$ Deutscher Bundestag, Stenografischer Bericht, 4. Sitzung, 15. Wahlperiode, 29.10.2002, S. 53; dipbt.bundestag.de/dip21/btp/15/15004.pdf [5.2.2020].

${ }^{25}$ Bill Clinton, Remarks at the World Trade Organization in Geneva, Switzerland, 18.5.1998; www.presidency.ucsb.edu/documents / remarks-the-world-trade-organization-geneva-switzerland [5.2.2020]. 
Fließen des Flusses oder dem Jahreszeitenwechsel, an denen man nichts ändern könne, bis hin zum Rad der Geschichte, das sich nicht zurückdrehen lasse - war der politische Diskurs einfallsreich. ${ }^{26}$ Bill Clinton sprach wiederholt von der „unerbittlichen Logik der Globalisierung “. ${ }^{27}$ Und Tony Blair traktierte seine Zuhörerinnen und Zuhörer mit Sätzen wie Hammerschläge:

\begin{abstract}
„Diese Kräfte des Wandels, die die Zukunft gestalten, machen nicht an nationalen Grenzen halt. Achten keine Tradition. Warten auf niemanden und auf keine Nation. Sie sind universell. Wir wissen, was ein Land des 21. Jahrhunderts braucht. Eine wissensbasierte Wirtschaft. Eine starke Zivilgesellschaft. Einen selbstbewussten Platz in der Welt. Sorge dafür, und ein Land wird die Zukunft meistern. Versäume es, und es wird das Opfer dieser Zukunft sein.“28
\end{abstract}

Die Rhetorik der Alternativlosigkeit beanstandeten politische Kritiker schon in der zeitgenössischen Debatte immer wieder. Ihr Vorwurf bezog sich aber, wie noch zu zeigen ist, weniger auf den Gedanken, die Globalisierung selbst sei unabänderlich. Denn diese Überzeugung teilten die allermeisten Kommentatoren, auch wenn sie mitunter abweichende Vorstellungen davon hatten, worin die Globalisierung bestehe. Die Kritik richtete sich gegen die politischen Lösungsstrategien, welche die Regierungsvertreter als unerlässlich ausgaben. Und darin lag tatsächlich der Fluchtpunkt der Bedrohungs- und Unausweichlichkeitsargumente: dass die Regierungen den richtigen Weg erkannt hätten, um die Gefahren zu entschärfen und den unabänderlichen Prozess in günstige Bahnen zu lenken. Viel Zeit blieb dafür allerdings nicht. Denn die beispiellose Rasanz der Ereignisse war ein weiteres integrales Element des Politikmodus, zu dem die Globalisierungsdiagnose Anlass gab. Die Regierungsvertreter führten den politischen Öffentlichkeiten emphatisch vor Augen, dass sich der aktuelle Wandel in einem Tempo vollziehe, das jedes bekannte Maß übersteige. Auf diese Weise evozierten sie einen Moment der Entscheidung: Die Weichen müssten jetzt gestellt werden, sonst sei es zu spät.

\title{
III. Sozialstaatsreformen als Imperativ der Globalisierung
}

Indem sich die Spuren des weltweiten Zusammenwachsens in nahezu allen Handlungsbereichen aufdecken ließen, verschaffte die Globalisierungsvorstellung den drei Regierungen ein in sich schlüssiges intellektuelles Fundament. Daraus ließen sich die politischen Zukunftsprogramme nahezu in Gänze ableiten. In der Innenpolitik zeitigte dies denkbar weitreichende Wirkungen. Die Ausgangssituationen, in der sich die neuen politischen Führungen fanden, wiesen dabei durchaus Ähn-

\footnotetext{
${ }^{26}$ Vgl. Deutscher Bundestag, Stenographischer Bericht, 44. Sitzung, 14. Wahlperiode, 16.6.1999, S. 3622 (Rezzo Schlauch, Bündnis 90/Die Grünen); dipbt.bundestag.de/dip21/btp/14/ 14044.pdf [10.2.2020].

${ }^{27}$ Clinton, Remarks on United States Foreign Policy, 26.2.1999.

28 Tony Blair, Leader's Speech, Bournemouth 1999; www.britishpoliticalspeech.org/speecharchive.htm?speech=205 [5.2.2020].
} 
lichkeiten auf. Für alle drei Länder waren die 1980er Jahre eine Zeit ökonomischer Prosperität gewesen, die indes durch steil ansteigende Staatsausgaben finanziert und - in den USA und Großbritannien stärker als in der Bundesrepublik - um den Preis wachsender sozialer Ungleichheit erkauft worden war. Das wirtschaftliche Wachstum hatte sich dann zu Beginn der 1990er Jahre abgeschwächt - und Massenarbeitslosigkeit erwies sich als endemisches Problem. Der Bundesrepublik erwuchs zudem aus der Finanzierung der deutschen Einheit eine kaum zu überschätzende zusätzliche Bürde. Vor diesem Hintergrund erschienen die Sozialstaatsausgaben überall als immer belastender und vor allem in den USA als vergleichsweise unwirksam, weil nur ein geringer Anteil der Bevölkerung davon profitierte; dies zeigte sich insbesondere in der Gesundheitsversorgung. Während die Clinton-Regierung sich schon seit Beginn der 1990er Jahre dezidiert um Abhilfe dieser strukturellen Probleme bemühte, entstand in der Bundesrepublik in den späten Jahren der Ära Kohl allenthalben der Eindruck eines „Reformstaus“. Dieser baute zusätzlichen Handlungsdruck auf, konnte aber auch der Legitimierung von Reformmaßnahmen dienen.

Die Vorhaben der Regierungen, die alle im Zeichen der inneren Erneuerung antraten, betrafen dann auch sehr ähnliche Bereiche, wenngleich sie durchaus unterschiedlich gelagert waren. Eine große Bedeutung kam den Reformen des Arbeitsmarkts und der Sozialhilfe zu. Die Clinton-Regierung stellte mehr Geld zur Verfügung, um Familien mit geringem Einkommen eine Kinderbetreuung zu ermöglichen. Ferner erhöhte sie den Mindestlohn, reduzierte die Steuerlast gering verdienender Arbeitnehmer und initiierte Berufsausbildungsprogramme. ${ }^{29}$ Dies war indes die Kehrseite drastischer Einschnitte: Der Personal Responsibility and Work Opportunity Reconciliation Act von 1996 beendete das auf Franklin D. Roosevelts New Deal zurückgehende Programm der Aid to Families with Dependent Children. Arbeitslose Eltern erhielten nun nach zwei Jahren keine finanzielle Unterstützung mehr und konnten eine solche über ihre gesamte Lebenszeit überhaupt nur fünf Jahre lang beanspruchen. Erwachsene ohne Kinder konnten Essensmarken nur noch alle drei Jahre für drei Monate erhalten, und legalen Einwanderern wurde die Wohlfahrtsunterstützung gestrichen.

New Labour band unter der Devise des „aktivierenden Sozialstaats“ die Sozialhilfe daran, dass die Empfänger Beschäftigungs- und Weiterbildungsangebote wahrnehmen würden. Blair brachte sogenannte Welfare-to-Work-Programme auf den Weg und führte den Mindestlohn sowie Steuerbegünstigungen für Bedürftige ein. ${ }^{30}$ Die rot-grüne Koalition in Deutschland stellte zunächst unter anderem die Altersvorsorge auf eine teilweise private Finanzierung um. Seit 2003 lancierte sie dann unter dem Schlagwort „Agenda 2010“ ein umfangreiches Programm von Veränderungen. Zahlreiche Maßnahmen von der Bezuschussung der Existenzgründung bis zur

\footnotetext{
${ }^{29}$ Vgl. Wilentz, Age; William H. Chafe, The Unfinished Journey. America Since World War II, Oxford ${ }^{8}$ 2015, S. 475-522, und Patrick J. Maney, Bill Clinton. New Gilded Age President, Lawrence 2016.

${ }^{30}$ Vgl. Seldon (Hrsg.), Blair's Britain, und Franz-Josef Brüggemeier, Geschichte Großbritanniens im 20. Jahrhundert, München 2010, S. 346-379.
} 
Lockerung des Kündigungsschutzes zielten darauf ab, den Arbeitsmarkt zu beleben. Ferner legte die Regierung Sozial- und Arbeitslosenhilfe zusammen, verringerte deren Bezugsdauer und erhöhte das Renteneintrittsalter. ${ }^{31}$ Auch auf anderen Politikfeldern ergaben sich Parallelen. Die Regierungen investierten in Bildung, die sie als entscheidende ökonomische Ressource definierten, und in die Gesundheitssysteme. Ebenso förderten Demokraten wie Labour und Rot-Grün die Telekommunikation und die Computerindustrie. Schließlich setzten sie die Deregulierung des Finanzsektors fort, die in den drei Ländern während der 1980er Jahre in Schwung gekommen war, wenn auch in unterschiedlichem Ausmaß.

Zwar gelang es keiner der Regierungen, alle ihre Erneuerungsvisionen umzusetzen. Gleichwohl sorgten sie dafür, dass Staat und Gesellschaft maßgeblich neu justiert wurden. Labour griff tief in wichtige gesellschaftliche Sektoren ein und veränderte damit in der einen oder anderen Weise das Leben zahlreicher Menschen ganz unmittelbar. Das Maßnahmenbündel der rot-grünen Regierung fügte sich sogar zur größten Sozialreform in der Geschichte der Bundesrepublik. Diese markierte zugleich das Ende des auf fortgesetzte Expansion ausgerichteten Sozialstaats, wie er spätestens nach dem Zweiten Weltkrieg errichtet worden war. Und auch wenn die Clinton-Regierung mit ihrem groß angelegten Vorhaben einer Reform der Gesundheitsversorgung schon früh scheiterte, hinterließen ihre späteren Reformen doch tiefe gesellschaftspolitische Spuren.

Am Beispiel der sozialstaatlichen Reformen und hier wiederum am deutschen Fall lässt sich besonders gut nachvollziehen, wie sehr sich dieser innere Umbau im Zeichen einer Globalisierungsdiagnose vollzog und wie sich diese genau auf das politische Handeln auswirkte. Die wirtschafts- und gesellschaftspolitische Neuausrichtung der rot-grünen Regierung stand unter der alles bestimmenden Prämisse, das eigene Land erfolgreich an die Realitäten einer globalisierten Welt anpassen zu müssen. Öffentlich rechtfertigte Kanzler Schröder etwa die Vorschläge der von Peter Hartz geleiteten Expertenkommission für die Arbeitsmarktreform mit den „Flexibilitätserfordernissen einer globalisierten Wirtschaft“. ${ }^{2}$ Das Thesenpapier für eine SPD-Klausurtagung im Januar 2003, das Edgar Wolfrum als die „Keimzelle der [...] Agenda 2010" bezeichnet hat, verwies auf die schwerwiegenden Folgen der Globalisierung. ${ }^{33}$ Und auch die „Agenda“ selbst wurde immer wieder damit begründet: „Die wirtschaftliche Globalisierung, die demographische Entwicklung und der technische Fortschritt", so führte Hubertus Heil für die SPD im Bundestag aus, „machen es dringend erforderlich, unseren Sozialstaat umzubauen, damit soziale Sicherheit auch in Zukunft möglich ist". ${ }^{34}$

Die Globalisierungsdynamik, die es aus der Sicht von Rot-Grün unabdingbar machte zu handeln, resultierte im Kern aus mehreren miteinander verschränkten

\footnotetext{
${ }^{31}$ Vgl. Wolfrum, Rot-Grün, und Herbert, Geschichte, S. 1231-1237.

${ }^{32}$ Deutscher Bundestag, Stenographischer Bericht, 248. Sitzung, 14. Wahlperiode, 4.7.2002, S. 25025; dipbt.bundestag.de/dip21/btp/14/14248.pdf [5.2.2020].

33 Wolfrum, Rot-Grün, S. 531.

${ }^{34}$ Deutscher Bundestag, Stenografischer Bericht, 56. Sitzung, 15. Wahlperiode, 3.7.2003, S. 4610; dipbt.bundestag.de/dip21/btp/15/15056.pdf [5.2.2020].
} 
Prozessen, auf welche die Politikerinnen und Politiker in ihren Bestandsaufnahmen immer wieder rekurrierten: dem Wachstum der internationalen Finanzmärkte, der steigenden Zahl und gleichzeitig zunehmenden geografischen Ausdehnung transnationaler Unternehmen und dem Aufschwung des grenzüberschreitenden Handels. All dies sei wiederum durch die Ausbreitung neuer Kommunikationsund Transporttechnologien ermöglicht worden. Das zentrale Reformargument, das daraus abgeleitet wurde, lautete, dass diese Entwicklungen einen zunehmenden Wettbewerb auf dem Weltmarkt erzeugten, der wiederum dazu zwinge, die Bedingungen des nationalen „Standorts“ (wie es jetzt zunehmend hieß) zu verbessern. Dafür müsste die Regierung sozialstaatliche Leistungen kürzen, Arbeitsschutzbestimmungen lockern und Unternehmensregulierungen abbauen. ${ }^{35}$

Mit diesem Reformdenken stand die Regierung in einem breiten diskursiven Bezugsfeld. So dürfte der vergleichsweise enge Austausch zwischen der deutschen, britischen und amerikanischen Regierung dazu beigetragen haben, dass sich manche Gemeinsamkeiten in den Problemwahrnehmungen und Lösungsstrategien abzeichneten. In dem als „Schröder-Blair-Papier“ bekanntgewordenen Plädoyer für Marktlogik, flexiblere Arbeitsformen und einen schlankeren Sozialstaat etwa bezogen sich die beiden Regierungschefs auch auf die Steuerungsvorgaben, die eine „Welt immer rascherer Globalisierung und wissenschaftlicher Veränderungen“ der Politik mache. ${ }^{36}$ Darüber hinaus jedoch war die Rezeptur, mit der RotGrün die deutsche Ökonomie stimulieren wollte, im Zuge der Hinwendung zu einer angebotsorientierten Wirtschafts- und Finanzpolitik schon seit den 1980er Jahren diskutiert worden. ${ }^{37}$ Seit Anfang der 1990er Jahre verschrieb sich ihr ein breites Geflecht nationaler und internationaler Akteure - angefangen von internationalen Finanzorganisationen wie der Organisation für wirtschaftliche Zusammenarbeit und Entwicklung, dem Internationalen Währungsfonds (IWF), der Weltbank und der 1995 gegründeten Welthandelsorganisation (World Trade Organization, WTO) über die Europäische Union (EU) und wirtschaftliche Interessenverbände bis hin zu einer Vielzahl ökonomischer Experten. Sie alle setzten in unter-

${ }^{35}$ Vgl. Colin Hay/Ben Rosamond, Globalization, European Integration and the Discursive Construction of Economic Imperatives, in: Journal of European Public Policy 9 (2002), S. $147-167$.

${ }^{36}$ Der Weg nach vorne für Europas Sozialdemokraten. Ein Vorschlag von Gerhard Schröder und Tony Blair, London, 8.6.1999; www.glasnost.de/pol/schroederblair.html [5.2.2020]. Vgl. auch Philip G. Cerny/Mark Evans, Globalisation and Public Policy under New Labour, in: Policy Studies 25 (2004), S. 51-65, und Wolfrum, Rot-Grün, S. 138-168.

${ }^{37}$ Vgl. Konrad H. Jarausch, Zwischen „Reformstau“ und „Sozialabbau“. Anmerkungen zur Globalisierungsdebatte in Deutschland, 1973-2003, in: Ders. (Hrsg.), Das Ende der Zuversicht? Die siebziger Jahre als Geschichte, Göttingen 2008, S. 330-352; Wencke Meteling, Nationale Standortsemantiken seit den 1970er-Jahren, in: Ariane Leendertz/Wencke Meteling (Hrsg.), Die neue Wirklichkeit. Semantische Neuvermessungen und Politik seit den 1970er-Jahren, Frankfurt a. M. 2016, S. 207-242, und Wencke Meteling, Standortsicherung = Zukunftssicherung. Zur angebotsorientierten Modellierung von Zukunft in der Standortdebatte, in: Christoph Kampmann/Angela Marciniak/Wencke Meteling (Hrsg.), „Security turns its eye exclusively to the future“. Zum Verhältnis von Sicherheit und Zukunft in der Geschichte, BadenBaden 2018, S. 389-420. 
schiedlichen Mischungsgraden auf den Abbau von Markt- und Handelshemmnissen, Steuererleichterungen und auf andere investitionsfördernde Maßnahmen, Entbürokratisierung sowie sozialpolitische Einschnitte. Auch die Regierung Kohl machte sich viele dieser Konzepte zu eigen, ohne sie allerdings in nennenswertem Maße umsetzen zu können.

In dieser Debatte war seit den späten 1980er Jahren zunächst noch eingeschränkt von den sich globalisierenden Märkten und vor allem Finanzmärkten die Rede gewesen. ${ }^{38}$ Mitte der 1990er Jahre wurde die Globalisierung dann im Sinne der sich wechselseitig verstärkenden, rapiden Zunahme der Finanzströme, des internationalen Handels und der ausländischen Direktinvestitionen als eine Art ökonomische Umweltbedingung mit weitreichenden Folgen identifiziert. ${ }^{39}$ Der IWF etwa setzte sich 1997 zentral mit der so verstandenen „Frage der Globalisierung“ auseinander, aus der sich für ihn die Forderung nach einer Politik ergab, „die den Erfordernissen kompetitiver Weltmärkte angepasst ist“. ${ }^{40}$ In der sogenannten Lissabon-Strategie von 2000 machte sich auch die EU diese Linie zu eigen. Sie präsentierte ihr ,ambitioniertes Programm für den Aufbau von Wissensinfrastrukturen, die Förderung von Innovation und Wirtschaftsreform und die Modernisierung der Sozialschutz- und Bildungssysteme“ als Reaktion auf den „Quantensprung [...], der aus der Globalisierung und den Herausforderungen einer neuen wissensbasierten Wirtschaft resultiert". ${ }^{41}$

In der Bundesrepublik fügte der Bundesverband der Deutschen Industrie den „Anpassungsdruck der Globalisierung“ 1996/97 prominent in sein seit Jahren gleichsam stehendes Plädoyer für eine Verbesserung der deutschen „Standortbedingungen“ ein, wodurch nun allerdings neue Begründungsfiguren hinzutraten. ${ }^{42}$ Die Globalisierung hatte demzufolge dem „weltweite[n] Konkurrenzkampf um Märkte und Marktanteile“ eine „ganz neue, vorher nicht gekannte Intensität“ verliehen. ${ }^{43}$ Der Sachverständigenrat zur Begutachtung der gesamtwirtschaftlichen Entwicklung nahm den Gedanken, dass die Globalisierung und von ihr ausgehend

\footnotetext{
${ }^{38}$ Zur Globalisierungsrede der späten 1980er Jahre vgl. Deutscher Bundestag, ,Jahresgutachten 1988/89 des Sachverständigenrates zur Begutachtung der gesamtwirtschaftlichen Entwicklung“, Bundestag-Drucksache 11/3478, 24.11.1988; www.sachverstaendigenrat-wirtschaft. de/fileadmin/dateiablage/download/gutachten/1103478.pdf [5.2.2020].

${ }^{39}$ Wichtige Stimuli stellten die Finanzkrise in Mexiko 1994 und die Asienkrise 1997/98 sowie dann die für viele überraschenden globalisierungskritischen Proteste 1999/2000 dar.

${ }^{40}$ International Monetary Fund, Annual Report 1997, Washington 1997, S. 24 f.; www.elibrary. imf.org/doc/IMF011/00315-9781451945102/00315-9781451945102/Other_formats/ Source_PDF/00315-9781455223350.pdf [19.3.2020].

${ }^{41}$ Europäischer Rat, Schlussfolgerungen des Vorsitzes, 23./24.3.2000; www.europarl.europa. eu/summits/lis1_de.htm [5.2.2020]. Zum Kontext vgl. Wirsching, Demokratie und Globalisierung, S. 73-88.

42 BDI-Bericht 1996, hrsg. vom Bundesverband der Deutschen Industrie, Köln 1997, S. 11. Insofern spricht einiges für das Argument Wencke Metelings, dass mit dem Globalisierungsargument zum Teil schon seit langem diskutierte Reform- und Umbauforderungen erst ihr durchschlagendes Gewicht erhielten; vgl. Meteling, Standortsemantiken, in: Leendertz/Meteling (Hrsg.), Neue Wirklichkeit.

${ }^{43}$ BDI-Bericht 1996, S. 8.
} 
der wachsende globale Wettbewerb die wirtschaftlichen Probleme in der Bundesrepublik verschärften, 1996 in sein Jahresgutachten auf und befasste sich zwei Jahre später eingehend mit diesem Zusammenhang. ${ }^{44}$ Seit der Jahrtausendwende verstand er die Globalisierung als prägende Gesamtbedingung, unter der die deutsche Wirtschaft zu operieren habe. ${ }^{45}$ Und auch die führenden deutschen Wirtschaftsforschungsinstitute begannen seit Mitte des Jahrzehnts, zunehmende Aufmerksamkeit auf Fragen der Globalisierung zu richten. Dabei standen die Informationsbedürfnisse der Regierung und die ökonomischen Forschungsanalysen in einer engen Wechselwirkung. So beauftragte das Bundeswirtschaftsministerium 1996/97 das Deutsche Institut für Wirtschaftsforschung in Berlin und das Kieler Institut für Weltwirtschaft damit, im Rahmen der sogenannten Strukturberichterstattung Aspekte der Globalisierung zu untersuchen. ${ }^{46}$ Überblickt man diese Fülle an Beiträgen, so wird erkennbar, dass die Bundesregierung an einem Konglomerat wirtschaftsbezogener Globalisierungsdiagnosen partizipierte, die sich kaum auf einen einzigen Ursprung zurückführen lassen. Vielmehr diffundierten sie im Zeitraum weniger Jahre gleichsam in alle Richtungen.

\section{Der unsichere Boden des (Nicht-)Wissens}

Doch wenngleich die Politiker und Ökonomen in den vorgeblich notwendigen Folgerungen, die sie aus der Globalisierung zogen, ein beträchtliches Maß an Gewissheit an den Tag legten, lagen die Dinge doch tatsächlich nicht so klar auf der Hand. Es war nicht ausgemacht, wie die globalen Veränderungen, die sie unablässig beschworen, beschaffen waren, wie dringlich sie es machten zu reagieren - und in welcher Weise. Auch in der analytischen Rückschau ist das keineswegs einfach

\footnotetext{
${ }^{44}$ Vgl. Deutscher Bundestag, „Jahresgutachten 1995/96 des Sachverständigenrates zur Begutachtung der gesamtwirtschaftlichen Entwicklung“, Bundestag-Drucksache, 13/3016, 15.11.1995, S. I-V und S. 172-190; www.sachverstaendigenrat-wirtschaft.de/fileadmin/ dateiablage/download/gutachten/1303016.pdf [5.2.2020], sowie Deutscher Bundestag, "Jahresgutachten 1997/98 des Sachverständigenrates zur Begutachtung der gesamtwirtschaftlichen Entwicklung“, Bundestag-Drucksache 13/9090, 18.11.1997, S. 170-184; www. sachverstaendigenrat-wirtschaft.de/fileadmin/dateiablage/download/gutachten/1309090. pdf [5.2.2020].

${ }^{45}$ Vgl. Deutscher Bundestag, ,Jahresgutachten 2000/01 des Sachverständigenrates zur Begutachtung der gesamtwirtschaftlichen Entwicklung“, Bundestag-Drucksache 14/4792, 29.11.2000, S. 180-188; www.sachverstaendigenrat-wirtschaft.de/fileadmin/dateiablage/ download/gutachten/00_ges.pdf [5.2.2020]. Eine sehr ähnliche Entwicklung zeigte der amerikanische Council of Economic Advisers, Report of the President, Washington 1990/ $1997 / 2000$.

${ }^{46}$ Vgl. Björn Alecke u. a., Neue Informations- und Kommunikationstechnologien, Tertiarisierung und Globalisierung - Herausforderungen für den Strukturwandel. Strukturberichterstattung 1996-1998, Bd. 2: Globalisierung und neue Informations- und Kommunikationstechnologien, München 1999; Jörn Kleinert u.a., Globalisierung, Strukturwandel und Beschäftigung, Tübingen 2000; Hans-Hagen Härtel u. a., Grenzüberschreitende Produktion und Strukturwandel. Globalisierung der deutschen Wirtschaft, Baden-Baden 1996, und Dietmar Edler u. a., Deutschland im Strukturwandel. Strukturberichterstattung 1997, Berlin 1998.
} 
zu bestimmen. Das absolute Wachstum der internationalen Finanzmärkte, des grenzüberschreitenden Warenaustauschs und der Zahl transnationaler Unternehmen, auf das die Zeitgenossen rekurrierten, war unleugbar rasant. ${ }^{47}$ Doch sagte es noch nichts darüber aus, wie stark die deutsche Wirtschaft verflochten war und was diese Verflechtungen bewirkten.

Der Komplex der ausländischen Investitionstätigkeit und der transnationalen Unternehmen, der in den politischen und ökonomischen Argumentationen eine Schlüsselrolle spielte, mag dies verdeutlichen. Denn erstens war der Anstieg ausländischer Direktinvestitionen, der als entscheidender Indikator für die zunehmende weltwirtschaftliche Integration galt, bereits als statistische Größe problematisch. Für sich genommen war er wenig aussagekräftig, denn der Verflechtungsgrad der Wirtschaft erhöhte sich nicht schon deshalb, weil die Direktinvestitionen zunahmen. Vielmehr musste man diese zu einer übergeordneten Größe in Beziehung setzen, was sich aber als schwierig erwies. Die meisten der Ökonomen, die sich um genauere Berechnungen bemühten, halfen sich damit, sie auf die Bruttoanlageinvestitionen zu beziehen; diese schlossen aber etwa den Kauf von Unternehmen nicht ein, während umgekehrt auch nicht alle Sachanlagen bei Auslandsgesellschaften durch Direktinvestitionen finanziert wurden. ${ }^{48}$ Wenn man aber dieses Verhältnis zugrunde legte, dann hatten ausländische Direktinvestitionen bis nach der Jahrhundertwende eine geringe Bedeutung. Weltweit machten sie im Zeitraum von 1990 bis 2003 gerade einmal acht Prozent der Bruttoanlageinvestitionen aus. ${ }^{49}$

Darüber hinaus war zweitens unklar, welche Effekte transnational organisierte Unternehmen auf die nationale Wirtschaft ausübten. Eine zeitgenössische Auswertung von Unternehmensdaten gelangte zu dem Schluss, dass die Wirtschaftstätigkeit multinationaler Firmen aus den USA, Großbritannien, Deutschland und Japan gemessen sowohl am Verkauf als auch am Vermögen zu Beginn der 1990er Jahre zu mehr als zwei Drittel auf die Herkunftsländer ausgerichtet war. ${ }^{50}$ Ferner flossen die weltweiten ausländischen Direktinvestitionen ganz überwiegend in hoch entwickelte Industrieländer und hier wiederum zu einem beträcht-

\footnotetext{
${ }^{47} \mathrm{Zu}$ ausländischen Direktinvestitionen vgl. United Nations Conference on Trade and Development (UNCTAD), Foreign Direct Investment. Inward and Outward Flows and Stock, Annual; unctadstat.unctad.org/wds/TableViewer/tableView.aspx?ReportId=96740 [5.2.2020], und zum Welthandel UNCTAD, Merchandise. Total Trade Growth Rates, Annual; unctadstat. unctad.org/wds/TableViewer/tableView.aspx?ReportId=102 [5.2.2020]. Berechnungen der Zahl transnationaler Unternehmen stützen sich zumeist auf den „World Investment Report“ der UNCTAD. Dessen Daten sind allerdings insofern unscharf, als er unter transnationalen Unternehmen solche versteht, die einen Anteil von zehn Prozent an ausländischen Unternehmen halten; vgl. UNCTAD, World Investment Report. Transnational Corporations, Extractive Industries and Development, S. 245; unctad.org/en/Docs/wir2007_en.pdf [5.2.2020]. Damit können sich jedoch sehr unterschiedliche geografische Reichweiten verbinden.

${ }^{48}$ Vgl. Härtel u. a., Produktion, S. 49.

${ }^{49}$ Vgl. Deutscher Bundestag, „Jahresgutachten 2004/05 des Sachverständigenrates zur Begutachtung der gesamtwirtschaftlichen Entwicklung“, Bundestag-Drucksache 15/4300, 18.11.2004, S. 365-370; dipbt.bundestag.de/dip21/btd/15/043/1504300.pdf [23.3.2020].

${ }^{50}$ Vgl. Paul Hirst/Grahame Thompson, Globalization in Question. The International Economy and the Possibilities of Governance, Cambridge 1996, S. 76-99.
} 
lichen Anteil in technologisch anspruchsvolle Branchen wie Chemie, Elektronik oder Autobau. Das mochte darauf verweisen, dass der vorrangige Grund für die Auslandsproduktion nicht in der Kostenersparnis lag.

Tatsächlich wiesen zeitgenössische Befragungen darauf hin, dass es Unternehmen bei ihrer Standortwahl in hohem Maße auf andere Faktoren wie die Nähe zum Absatzmarkt, politische Stabilität, hochwertige Infrastruktur und ein hohes Ausbildungsniveau ankam. ${ }^{51}$ Für die Bundesrepublik bedeuteten diese Befunde zum einen, dass der nationale Wirtschafts-Standort trotz vergleichsweise hoher Lohnkosten und Unternehmenssteuern nicht notwendigerweise ungünstig sein musste. Zum anderen trug die Auslandsproduktion deutscher Unternehmen, sofern es ihr gar nicht darum ging, billige Arbeitskräfte zu gewinnen, nicht unbedingt zum heimischen Beschäftigungsproblem bei. In der wirtschaftswissenschaftlichen Diskussion nahmen die Stimmen eher zu, die der unternehmerischen Aktivität im Ausland positive gesamtwirtschaftliche Folgen attestierten, da sie dazu angetan sei, die deutsche Exporttätigkeit zu stärken. ${ }^{52}$ In einem bemerkenswerten Bericht von 2004 schloss sich auch der Sachverständigenrat dieser Sicht an. Damit widerlegte er ebenso stillschweigend wie ungerührt eine der zentralen Befürchtungen, mit der er selbst seit Mitte der 1990er Jahre die Forderung nach Deregulierungen und Lohnsenkungen motiviert hatte. ${ }^{53}$

Schließlich ließ sich fragen, ob die immer wieder ins Feld geführten Wachstums- und Verknüpfungsprozesse mit dem Begriff Globalisierung überhaupt treffend beschrieben waren. Weltweit betrachtet, konzentrierten sich die ausländischen Direktinvestitionen - wie auch der grenzüberschreitende Handel zwischen Mitte der 1990er und Mitte der 2000er Jahre auf den amerikanisch-europäisch-japanischen Raum. ${ }^{54}$ Die Bundesrepublik war und blieb in eine Wirtschaftszone eingebunden, die im Wesentlichen aus Europa und den USA bestand. So vereinigten die Länder der EU, die Schweiz und die USA zusammengenommen im Jahr 1998 fast 80 Prozent der deutschen Direktinvestitionen im Ausland und mehr als 90 Prozent der ausländischen Direktinvestitionen in Deutschland auf sich; im Jahr 2005 waren diese Zahlen auf 88 beziehungsweise fast 94 Prozent gestiegen. ${ }^{55}$

${ }^{51}$ Vgl. Geoffrey Garrett/Deborah Mitchell, Globalization, Government Spending and Taxation in the OECD, in: European Journal of Political Research 39 (2001), S. 145-177; Kleinert u. a., Globalisierung, S. 204-211, und Alecke u. a., Informations- und Kommunikationstechnologien, Bd. 2, S. 161-170.

${ }^{52}$ Vgl. Kleinert u. a., Globalisierung.

${ }^{53}$ Vgl. Deutscher Bundestag, „Jahresgutachten 2004/05“, Bundestag-Drucksache 15/4300, 18.11.2004, S. 47-50 und S. 349-386.

${ }^{54}$ Vgl. UNCTAD, World Investment Report 1995. Transnational Corporations and Competitiveness; unctad.org/en/Docs/wir1995overview_en.pdf; UNCTAD, World Investment Report 1998. Trends and Determinants; unctad.org/en/Docs/wir1998overview_en.pdf, und UNCTAD, World Investment Report 2006. FDI from Developing and Transition Economies: Implications for Development; unctad.org/en/Docs/wir2006overview_en.pdf. Alle Links wurden am 5.2.2020 das letzte Mal aufgerufen.

${ }^{55}$ Vgl. Deutsche Bundesbank, Deutsche Direktinvestitionen im Ausland und ausländische Direktinvestitionen in Deutschland nach Bundesländern, 11.4.2005; www.bundesbank.de/ resource/blob/649916/7158830431f9ab07165542717be3fa4f/mL/direktinvestitionen-1998- 
Nur an diesem Indikator gemessen, nahm die seit Jahrzehnten überkommene europäisch-transatlantische Ausrichtung der deutschen Wirtschaft um die Jahrhundertwende also noch zu. Der semantische Überschuss des Globalisierungsbegriffs dagegen erweckte die Vorstellung, alle wirtschaftlichen Entwicklungen auf der Welt seien für die nationalen Volkswirtschaften plötzlich ganz unmittelbar relevant, und sorgte damit für eine geografische Entgrenzung.

Bei näherem Hinsehen ließ (und lässt) sich mithin der Ausgangsbefund, mit dem die rot-grüne Regierung wie auch viele Experten die weitreichenden Sozialund Arbeitsmarktreformen wesentlich begründeten, von verschiedenen Seiten aus in Frage stellen. Die stehende wirtschaftspolitische Prämisse der 1990er und frühen 2000er Jahre, die „Globalisierung der Märkte“ zwinge zu „Anpassungen“, verdeckte sehr viel mehr ökonomische Zusammenhänge, als sie offenlegte. Es wäre sicherlich übertrieben zu behaupten, dass die Rede von den globalisierten Märkten ähnlich aussagekräftig war, als hätten die Politiker und Ökonomen auf Planetenkonstellationen oder den Vogelflug am Himmel verwiesen. Stark übertrieben wäre es aber nicht. Denn auf den nachprüfbaren Kerngehalt reduziert, war damit nicht mehr ausgesagt, als dass man Veränderungen beobachten könne, die irgendeine Bedeutung schon haben würden. In der öffentlichen Rede, zum Teil aber auch in den Berichten und Empfehlungen der Wirtschaftsexperten gewann der Globalisierungsbegriff den Status einer Kurzformel für denkbar komplexe wirtschaftliche Sachverhalte, die nicht einmal ansatzweise hergeleitet und entfaltet, sondern lediglich postuliert wurden.

Die zeitgenössischen Versuche, das Phänomen Auslandsproduktion zu verstehen, deuten bereits an, dass das Nachdenken über die Folgen der Globalisierung keineswegs monolithisch war. In der Bundesrepublik (und darüber hinaus) entfaltete sich eine intensive Debatte, in der durchaus abweichende Einschätzungen vorgebracht wurden. So bildete sich um die Mitte der 1990er Jahre eine zurückhaltende bis skeptische Position heraus, wenngleich sie mit den Jahren eher schwächer wurde. Eine Reihe von Ökonomen etwa hielt die weltwirtschaftlichen Veränderungen für geografisch so eingegrenzt, dass sich nicht von einer Globalisierung sprechen lasse, sah zumal die deutsche Wirtschaft nicht als globalisiert an und beurteilte schließlich den Effekt der weltwirtschaftlichen Wandlungen als nicht so gravierend, wie es in der politischen Diskussion erscheinen mochte. ${ }^{56}$ In den zugrundeliegenden Denkfiguren unterschieden sich diese Experten insofern von den Verfech-

\footnotetext{
data.pdf [5.2.2020], und Deutsche Bundesbank, Deutsche Direktinvestitionen im Ausland und ausländische Direktinvestitionen in Deutschland nach Bundesländern, 30.4.2008; www.bundesbank.de/resource/blob/649748/c0b5e005962fef068292158392b644b0/mL/ direktinvestitionen-2005-data.pdf [5.2.2020].

${ }^{56}$ Vgl. Härtel u. a., Produktion; Hans-Hagen Härtel/Rolf Jungnickel, Strukturprobleme einer reifen Volkswirtschaft in der Globalisierung. Analyse des sektoralen Strukturwandels in Deutschland, Baden-Baden 1998, und Harald Trabold, Zum Verhältnis von Globalisierung und Sozialstaat, in: Aus Politik und Zeitgeschichte B 48/2000, S. 23-30. Als orthodoxe Positionen vgl. Peter Nunnenkamp u. a., Globalisation of Production and Markets, Tübingen 1994; Edler u. a., Deutschland, und Hans-Werner Sinn, Wie viel Globalisierung verträgt die Welt?, in: Ifo Schnelldienst 55 (2002), S. 3-16.
} 
terinnen und Verfechtern globalistischer Reformzwänge, als sie sich um Disaggregierung und Differenzierung bemühten, wo jene Zusammenhänge herstellten.

Auch die Sozialwissenschaften setzten sich eingehend mit diesen Fragen auseinander. Ein Forschungsstrang plädierte ganz unmittelbar dafür, den Zusammenhang von Globalisierung und Sozialstaat stärker in seine Einzelteile zu zerlegen. ${ }^{57}$ Dabei wurde der Gedanke formuliert, die Globalisierung sei nicht als ökonomischer Fundamentalprozess zu begreifen, der die gleichsam externe Bedingung anderer politischer und sozialer Phänomene darstelle. Stattdessen gehe es darum zu verstehen, welche Teilprozesse der Globalisierung sich auf welche Bereiche der Wirtschaft auswirkten. Dadurch entstand ein vielschichtiges Bild - oder dieses wurde doch immerhin angedeutet - demzufolge partielle und insgesamt eher schwache Zusammenhänge zwischen der weltwirtschaftlichen Verflechtung und sozialstaatlichen Gegebenheiten bestanden.

Aus derartigen, um feinanalytische Nuancen bemühten Bestandsaufnahmen wurden indes wiederum unterschiedliche Handlungsvorschläge abgeleitet, so dass zwischen den Ausgangsbefunden und den wirtschaftspolitischen Positionen letztlich kein fester Zusammenhang bestand. Gerade diejenigen Ökonomen, die die Globalisierungsdynamik für nicht sehr ausgeprägt hielten, wiesen darauf hin, dass sie gleichwohl den internationalen Standortwettbewerb verschärfe und es deshalb geboten sei, den deutschen Arbeitsmarkt zu flexibilisieren (wie der ubiquitäre Begriff lautete), die Lohnkosten zu senken und das Ausbildungsniveau zu verbessern. ${ }^{58}$ Das hatte zum Teil mit einem prognostischen Vorausblick zu tun. Denn einige der nüchternen Stimmen - aber etwa auch der Bundesverband der Deutschen Industrie machten geltend, dass die nationale (und internationale) Wirtschaft zwar noch nicht weltweit verflochten sei, die Entwicklungen, die in diese Richtung wiesen, aber gerade jüngst stark zugenommen hätten. ${ }^{59}$ Damit gewann die Argumentation ein antizipatorisches Element, ging es doch um eine Zukunft, die unmittelbar bevorstand.

In der politischen Diskussion dagegen wurden die disaggregierenden Einschätzungen der Sozial- und Wirtschaftswissenschaften auch aufgegriffen, um damit politische Gegenpositionen zu stützen. Das traf zuvorderst auf den SPD-Vorsitzenden

${ }^{57}$ Vgl. John Clarke, Globalization and Welfare States. Some Unsettling Thoughts, in: Robert Sykes/Bruno Palier/Pauline M. Prior (Hrsg.), Globalization and European Welfare States. Challenges and Change, Houndmills 2001, S. 19-37; Garrett/Mitchell, Globalization; Brian Burgoon, Globalization and Welfare Compensation. Disentangling the Ties that Bind, in: International Organization 55 (2001), S. 509-551, und Philipp Genschel, Globalization and the Welfare State. A Retrospective, in: Journal of European Public Policy 11 (2004), S. 613-636. Eine wichtige Diskussion entspann sich ferner um die Frage der Konvergenz der Nationalstaaten, die allerdings auf einer anderen Ebene lag; vgl. Sykes / Palier/Prior (Hrsg.), Globalization; Wolfgang Merkel u. a., Die Reformfähigkeit der Sozialdemokratie. Herausforderungen und Bilanz der Regierungspolitik in Westeuropa, Wiesbaden 2006, und Reimut Zohlnhöfer, Globalisierung der Wirtschaft und finanzpolitische Anpassungsreaktionen in Westeuropa, Baden-Baden 2009.

${ }^{58}$ Vgl. Härtel u. a., Produktion, S. 32, und Kleinert u. a., Globalisierung, S. 211.

${ }^{59}$ Vgl. Marianne Beisheim u. a., Im Zeitalter der Globalisierung? Thesen und Daten zur gesellschaftlichen und politischen Denationalisierung, Baden-Baden 1999, und Deutscher Bundestag, ,Jahresgutachten 2004/05“, Bundestag-Drucksache 15/4300, 18.11.2004. 
Oskar Lafontaine zu. In einer 1998 erschienenen Schrift, die er zusammen mit Christa Müller verfasst hatte, wandte sich auch Lafontaine gegen die Vorstellung, die deutsche Wirtschaft sei globalisiert. ${ }^{60}$ Das galt in seiner Sicht zwar für die Finanzmärkte, nicht aber für Handel und Arbeitsmarkt. Daher sprach er sich gegen die Senkung von Löhnen, Sozialleistungen und Unternehmenssteuern aus und kennzeichnete damit die Position, die ihn nach der Bundestagswahl in zunehmenden Gegensatz zu den „Modernisierern“ um Bundeskanzler Schröder manövrieren sollte. Andere Vorschläge Lafontaines bewegten sich aber durchaus auf der Linie der überwiegend für notwendig gehaltenen Rezepte. Denn auch er bekannte sich zum Wettbewerb der Unternehmen und Staaten, den er indes über eine bessere Produktivität ausgetragen wissen wollte, sowie zu Investitionen in Bildung und Infrastruktur.

Nimmt man die Diskussion über den Konnex von Globalisierung und sozialpolitischen Reformimpulsen als Ganzes und blendet den Gestus der Unfehlbarkeit aus, den mehr oder minder alle Seiten zur Schau stellten, so wird deutlich, dass die politischen Entscheidungen der rot-grünen Regierungsjahre in einer Situation der äußersten Ungewissheit getroffen wurden. Viele postulierte Zusammenhänge wurden dicht belegt, doch wiesen sie in ganz unterschiedliche Richtungen, und mögen manche auch wahrscheinlicher gewesen sein als andere, so blieb doch keiner unbestritten. Letztlich glich die Diskussion einem Stochern im Nebel, wobei sich schlicht nicht zuverlässig sagen ließ, welche wirtschafts- und sozialpolitischen Notwendigkeiten aus den weltweiten Verflechtungen hervorgingen. Von hier aus betrachtet, wird noch einmal besonders klar fassbar, dass die Globalisierung nicht der Wandel war, der sich in den 1990er und 2000er Jahren vollzog, sondern dass die Globalisierungsdiagnose einen Modus darstellte, diesen Wandel zu verarbeiten.

\section{Die Überzeugungskraft der All-Erklärung}

Zugleich werfen diese Beobachtungen die Frage auf, warum die Vorstellung eines Konnexes zwischen Globalisierung und sozialstaatlichen Leistungsproblemen zumindest in maßgeblichen politischen und Expertenkreisen eine so große Wirkmacht erlangte. Zeitgenössisch bildete sich eine kräftige Argumentationslinie heraus, die besagte, die Globalisierungsrede sei eine Strategie, mit der von unerfreulichen Wahrheiten abgelenkt oder sogar eine radikal liberale Wirtschaftsideologie durchgesetzt werden solle. In einem Strang der soziologischen Forschung wurde diese Sicht wissenschaftlich - und folglich mit einer weniger offensichtlich politischen Aufladung - reproduziert. Nach der Jahrtausendwende verlegten sich Sozialwissenschaftlerinnen und -wissenschaftler darauf, Globalisierung als einen Diskurs zu untersuchen, wobei sie praktisch ausschließlich auf dessen taktisch-instrumentelle und legitimierende Funktion abhoben. ${ }^{61}$ In der Parteienlandschaft

\footnotetext{
${ }^{60}$ Vgl. Oskar Lafontaine/Christa Müller, Keine Angst vor der Globalisierung. Wohlstand und Arbeit für alle, Bonn 1998.

${ }^{61}$ Vgl. Sykes/Palier/Prior (Hrsg.), Globalization; zu New Labour vgl. Daniel T. Dye, New Labour, New Narrative? Political Strategy and the Discourse of Globalisation, in: The British
} 
der rot-grünen Regierungsjahre war es vor allem die Partei des Demokratischen Sozialismus, die diesen Diskurs lautstark beklagte. Dass der „Sachzwang der Globalisierung“ als „Entschuldigung für neoliberale Wirtschaftspolitik“ eingesetzt werde, avancierte in den einschlägigen Debatten zum ceterum censeo der Partei. ${ }^{62}$ Allerdings hatte in den Jahren vor dem Regierungswechsel auch der linke Flügel der SPD den Vorwurf an die Regierung Kohl gerichtet, „die neue Zauberformel der Globalisierung schamlos und einseitig [zu] mißbrauchen, um Löhne zu drücken, Sozialstandards zu reduzieren und die Arbeitnehmer insgesamt öffentlich zu diffamieren". ${ }^{63}$ Und in der Oppositionsrolle ließ es sich wiederum die CDU nicht nehmen, die neue Regierung zu beschuldigen, sie mache die Globalisierung „zur Ausrede oder zum Fluchtweg für die innen-, wirtschafts- und sozialpolitischen Probleme in diesem Lande“. ${ }^{64}$

Die Anschuldigungen, die im linken parteipolitischen Spektrum laut wurden, teilte die außerparlamentarische Bewegung der sich selbst so titulierenden Globalisierungskritiker, die in der Bundesrepublik seit dem Jahr 2000 aufkam. International hatte sich diese Bewegung angesichts der asiatischen Währungs- und Wirtschaftskrise 1997/98 im Protest gegen die wahrgenommene Macht der Finanzmärkte formiert. Fortan richtete sie sich gegen die als neoliberal stigmatisierte Weltwirtschaftsordnung und deren Repräsentanten. ${ }^{65}$ Wie andernorts bemängelten die globalisierungskritischen Gruppen auch in Deutschland die von kapitalistischen Profitinteressen vorangetriebene Zerstörung einer lebenswerten Welt. Deren Symptome erkannten sie in der wachsenden Kluft zwischen Arm und Reich weltweit wie auch in den Industrieländern, in Umweltzerstörung, der Aushöhlung der Demokratie durch den Einfluss unternehmerischer Entscheidungen und in

Journal of Politics and International Relations 17 (2015), S. 531-550; Czerny/Evans, Globalisation; Emilie L'Hôte, New Labour and Globalization. Globalist Discourse with a Twist?, in: Discourse and Society 21 (2010), S. 355-376; Holger Rossow, Globalismus und New Labour. Zur diskursiven Konstruktion von Globalisierungsprozessen im Großbritannien der Blair-Ära, Bielefeld 2011; Robert H. Cox, The Social Construction of an Imperative. Why Welfare Reform Happened in Denmark and the Netherlands but Not in Germany, in: World Politics 53 (2001), S. 463-498, und Vivien A. Schmidt, Does Discourse Matter in the Politics of Welfare State Adjustment?, in: Comparative Political Studies 35 (2002), 168-193.

${ }^{62}$ Deutscher Bundestag, Stenographischer Bericht, 16. Sitzung, 14. Wahlperiode, 21.1.1999, S. 1052 (Ursula Lötzer, PDS); dipbt.bundestag.de/dip21/btp/14/14016.pdf [5.2.2020].

${ }^{63}$ Deutscher Bundestag, Stenographischer Bericht, 117. Sitzung, 13. Wahlperiode, 28.6.1996, S. 10608 (Ottmar Schreiner, SPD); dip21.bundestag.de/dip21/btp/13/13117.pdf [26.3. 2020].

${ }^{64}$ Deutscher Bundestag, Stenographischer Bericht, 44. Sitzung, 14. Wahlperiode, 16.6.1999, S. 3611 (Wolfgang Schäuble, CDU / CSU).

${ }^{65}$ Vgl. Dieter Rucht/Roland Roth, Globalisierungskritische Netzwerke, Kampagnen und Bewegungen, in: Roland Roth/Dieter Rucht (Hrsg.), Die sozialen Bewegungen in Deutschland seit 1945. Ein Handbuch, Frankfurt a. M./New York 2008, S. 493-512; Donatella della Porta/Dieter Rucht (Hrsg.), Meeting Democracy. Power and Deliberation in Global Justice Movements, Cambridge 2013; Michelle Beyeler, Was bewirkt Globalisierungskritik? Protestkampagnen gegen die Welthandelsorganisation und das Weltwirtschaftsforum, Frankfurt a. M./New York 2013, und Christine Unrau, Erfahrung und Engagement. Motive, Formen und Ziele der Globalisierungskritik, Bielefeld 2018. 
kultureller Nivellierung. ${ }^{66}$ Schon bald verlegten sich die deutschen Globalisierungskritikerinnen und -kritiker indes auch auf den Protest gegen die rot-grüne Sozialpolitik. Die „Agenda 2010“ verurteilten sie als „knallharte[n] Neoliberalismus“. ${ }^{67}$ In ihren Augen agierte die Bundesregierung als Erfüllungsgehilfin der Finanzinteressen transnationaler Konzerne, die sie im Verbund mit anderen westlichen Regierungen und den von ihnen gelenkten internationalen (Finanz-)Organisationen zielgerichtet durchsetzte.

Dass in der Globalisierungsrede der Regierungen instrumentelle Motive eine Rolle spielten, ist auch in der Rückschau nicht von der Hand zu weisen. Wenn die führenden Politiker die Globalisierung ins Spiel brachten, konnten sie wirtschaftliche Probleme einem vermeintlich äußerlich gegebenen, nicht selbst zu verantwortenden Prozess zurechnen und dadurch unbequeme Maßnahmen abfedern. Eine ausreichende historische Erklärung für die politische Prägekraft der Globalisierungsvorstellung liefert dies aber nicht, denn auch strategische Argumente müssen plausibel erscheinen, um zu verfangen oder zumindest als aussichtsreich zu gelten. Der Vorwurf des neoliberalen Herrschaftsdiskurses wiederum fand Anknüpfungspunkte darin, dass sich in den Einschätzungen der internationalen Finanzorganisationen, der westlichen Regierungen und der Experten-Communities tatsächlich eine wirtschaftspolitische Orthodoxie herausbildete, die auf fortgesetzte Liberalisierung und Deregulierung drängte. Allerdings setzte die globalisierungskritische Sicht die ziemlich heterogenen Interessenlagen einer Vielzahl von Akteuren stark vergröbernd in eins. Das reichte nicht selten soweit, dass das Bild einer Art Weltschattenregierung evoziert wurde, die im Verborgenen operierte, wo sie von einem subjektlosen Finanzkapital gesteuert wurde. ${ }^{68}$ Und selbst dann erfasste die Kritik allein den zweifellos dezidierten Glauben an die Vorzüge einer marktgesteuerten, vernetzten Wirtschaft und damit nur eine Facette des Globalisierungsdenkens, wie es die Politik der westlichen Regierungen leitete.

Tatsächlich entwickelte der Globalisierungsgedanke eine immense Überzeugungskraft, die weit über jedes instrumentelle Kalkül hinausreichte. Das zeigte sich nicht zuletzt darin, dass nahezu niemand grundsätzlich aus den diskursiven Bahnen ausscherte. ${ }^{69}$ Selbst die um Differenzierung bemühten Sozialwissenschaftler und Ökonomen teilten den Konsens, dass es die Globalisierung gebe, dass sie etwas bewirke, und dass sie mit den sozialstaatlichen Problemen in einem wie auch immer gearteten Zusammenhang stehe. Unabhängig davon, wie sie sich inhaltlich positionierten, verliehen sie mit ihren Studien sogar dem Gedanken Nachdruck, man könne die Globalisierung exakt messen (wenn man nur komplex genug rechnete). Die Globalisierungskritikerinnen und -kritiker ihrerseits legten viel Empha-

\footnotetext{
${ }^{66}$ Vgl. Grundsätze von Attac Deutschland, in: Alles über Attac, hrsg. von Attac Deutschland, Frankfurt a. M. 2004, S. 112-116.

${ }^{67}$ Peter Wahl, Standpunkte und Perspektiven, in: Ebenda, S. 23-34, hier S. 25.

${ }^{68}$ Vgl. Christiane Grefe/Mathias Greffrath/Harald Schumann, Attac. Was wollen die Globalisierungskritiker?, Berlin 2002, S. 9.

${ }^{69}$ Vgl. Linda Weiss, Globalization and the Myth of the Powerless State, in: New Left Review 225 (1997), S. 3-27, und Hirst/Thompson, Globalization in Question.
} 
se in die Beteuerung, nicht die Globalisierung als solche abzulehnen, sondern allein ihre neoliberale Variante, während sie eine andere Form der Globalisierung anstrebten..$^{70}$ Mitunter stimmten sie mit den Analysen der Politiker und Experten bis in die Details überein: Der „Abbau des Wohlfahrtsstaates“ war „nur im Zusammenhang mit der Globalisierung zu verstehen“, da er letztlich auf den „grenzüberschreitenden Handel mit Waren, Dienstleistungen und Kapital“ zurückging. ${ }^{71}$ Das sah die rot-grüne Regierung nicht um ein Haar anders. Worin sich beide unterschieden, waren die politische Bewertung und die für geboten erachteten Konsequenzen - darin allerdings erheblich.

Die Globalisierungsvorstellung bezog ihre suggestive Evidenz daraus, dass sie bei dem lebensweltlichen Veränderungskomplex ansetzte, den die Zeitgenossinnen und Zeitgenossen der Jahrtausendwende am meisten und als geradezu radikal neuartig wahrnahmen - den Abbau von Barrieren und die Ausdehnung der räumlichen Bezüge - und ihn ins Zentrum des Gegenwartsverständnisses rückte. Abstrakter formuliert wurde die Perzeption des Neuen in ein starkes Kausalitätsprinzip übersetzt. Dieses Prinzip war sowohl offen als auch gerichtet. So erlaubte es das Globalisierungsdenken, zahlreiche als gravierend angesehene zeitgenössische Entwicklungen miteinander in Verbindung zu bringen und gleichzeitig als Teil einer überwölbenden Wandlungsdynamik zu begreifen. Wer sich darauf einließ, gewann ein politisches Welterklärungsmodell mit hoher Binnenkohärenz - ganz unabhängig von der jeweiligen politischen Haltung. Dieses Modell war das Resultat einer intensiven Suche nach großen Zusammenhängen, denn als solche stellt sich die Globalisierungsdebatte aus der historischen Distanz betrachtet insgesamt wie auch in ihrem sozialstaatlichen Teilbereich dar. Es füllte das beschriebene Vakuum, das entstanden war, als die ideologischen Großentwürfe, die während des 20. Jahrhunderts lange Zeit feste Einordnungen des innen- und weltpolitischen Geschehens ermöglicht hatten, ihre Erklärungskraft verloren.

Im Ergebnis scheint die Politik der drei Regierungen dazu beigetragen zu haben, die Globalisierung, so wie sie sie verstanden und argumentativ einsetzten, dramatisch zu befördern. Am Beispiel der amerikanischen Freihandelspolitik lässt sich das genauer studieren. Aufbauend auf den eher vereinzelten Ansätzen der Regierung George Bushs, machte die Clinton-Regierung die Durchsetzung des Freihandels zu einer tragenden Säule ihres Versuchs, die stockende amerikanische Ökonomie wiederzubeleben. Mit dem emphatischen Verweis darauf, dass die globale Wirtschaft mit immenser Geschwindigkeit zusammenwachse und daraus ein harter weltweiter Wettbewerb entstehe, in dem es darauf ankomme, „to compete and to win“, wurde die amerikanische Regierung zum entschiedensten Befürworter weitreichender internationaler Vereinbarungen. ${ }^{72}$ Auch wenn längst nicht alle Initiativen erfolgreich waren, wuchs das Netz an Freihandelsvereinbarungen im-

\footnotetext{
${ }^{70}$ Vgl. Naomi Klein, Über Zäune und Mauern. Berichte von der Globalisierungsfront, Frankfurt a. M. 2003.

71 Wahl, Standpunkte, S. 26.

72 Zur ersten großen programmatischen Rede zum Freihandel Clintons, vgl. ders., Remarks at the American University Centennial Celebration, 26.2.1993; www.presidency.ucsb.edu/
} 
mens. ${ }^{73}$ Als besonders bedeutsam erwies sich das (von Bush auf den Weg gebrachte) North Atlantic Free Trade Agreement, das die Clinton-Regierung 1993 abschloss. Zugleich bemühte sie sich in der sogenannten Uruguay-Runde um Handelsliberalisierungen im Rahmen des General Agreement on Tariffs and Trade und anschließend in der neu geschaffenen Welthandelsorganisation. Ferner resultierte die amerikanische Politik 1994 in Absichtserklärungen über die Schaffung einer Freihandelszone der Asiatisch-Pazifischen Wirtschaftsgemeinschaft (die bis 2000 wenig Fortschritt machen sollte) und einer Amerikanischen Freihandelszone (über die 1998 formale Verhandlungen aufgenommen wurden). Schließlich traf die Clinton-Regierung 1999 eine bilaterale Vereinbarung mit Japan und 2000 eine mit China. Die damit hergestellten „dauerhaft normalen Handelsbeziehungen“ bahnten der Volksrepublik den Weg in die WTO. Innenpolitisch war sie heftig umstritten, weil Clinton dafür die Kopplung zwischen Chinas Meistbegünstigungsstatus und menschenrechtlichen Verbesserungen im Land wieder zurücknehmen musste, die er im Mai 1993 mit Aplomb verkündet hatte.

In ihrer Summe katapultierte diese Politik den amerikanischen Außenhandel in neue Dimensionen; am Ende von Clintons Amtszeit hatte sich sein Gesamtvolumen gegenüber 1992 fast verzwanzigfacht. Schon 1994 war der Durchschnittswert der 1980er Jahre - wie sich zeigen sollte, dauerhaft - überschritten. ${ }^{74}$ Die Außenhandelsquote, die im Durchschnitt der 1980er Jahre bei etwas über 18 Prozent gelegen hatte, war in diesem Zeitraum von fast 20 auf knapp 25 Prozent gestiegen, so dass sich auch die relative Bedeutung des Außenhandels für die amerikanische Wirtschaft merklich erhöht hatte. ${ }^{75}$ Auf diesem Feld zeigte sich mithin besonders zugespitzt, wie die Regierungen die Bedingungen der Globalisierung, auf die sie behaupteten, reagieren zu müssen, durch die Maßnahmen, die sie dafür ergriffen, selbst schaffen konnten. Auch bei einer zurückhaltenden Beurteilung fällt es schwer, dieser Politik nicht Züge einer Self-Fulfilling Prophecy zu attestieren. ${ }^{76}$ Die

node/220231 [10.2.2020]. Vgl auch ders., Remarks on the House of Representatives Action on the North American Free Trade Agreement, 17.11.1993, hier das Zitat.

${ }^{73}$ Vgl. Robert Z. Lawrence, International Trade Policy in the 1990s, in: Jeffrey Frankel/Peter Orszag (Hrsg.), American Economic Policy in the 1990s, Cambridge 2002, S. 277-323; Gary Clyde Hufbauer/Jeffrey J. Schott, NAFTA Revisited. Achievements and Challenges, Washington 2005, und Mickey Kantor, Adapting to a Changing Global Economy, in: Richard Himelfarb/Rosanna Perotti, A True Third Way? Domestic Policy and the Presidency of William Jefferson Clinton, New York 2014, S. 33-37.

${ }^{74}$ Die Berechnungen nach den Zahlen U. S. Census Bureau, U. S. Trade in Goods and Services. Balance of Payments Basis, 6.3.2020, Stand: 23.3.2020; www.census.gov/foreign-trade/ statistics/historical/grands.pdf [23.3.2020].

${ }^{75}$ Bis 2011 wuchs die Außenhandelsquote weiter bis auf fast 31 Prozent, bevor sie wieder zurückging. Berechnungen nach Statista, Imports of Goods and Services in the United States from 1990 to 2017, as a percentage of GDP; www.statista.com/statistics /259096/us-imports-as-apercentage-of-gdp/; Exports of Goods and Services from the United States from 1990 to 2017, as a Percentage of GDP; www.statista.com/statistics /258779/us-exports-as-a-percentageof-gdp/; vgl. auch: www.theglobaleconomy.com/USA/Imports / und www.theglobaleconomy. com/USA/Exports /. Alle Links wurden am 10.2.2020 das letzte Mal aufgerufen.

${ }^{76}$ Zur britischen Politik vgl. Hay/Rosamond, Globalization. 
antizipierte Zukunft war nun eingetreten - nachdem maßgebliche Akteure nach Kräften darauf hingearbeitet hatten.

\section{Globalisierung als außenpolitische Ermächtigung}

Bei alledem leiteten die drei Regierungen nicht nur die innere Neuausrichtung aus den Imperativen der Globalisierung her, sondern ebenso ihre internationale Positionierung. Dieser Nexus wurde in den politischen Öffentlichkeiten offenbar sehr viel weniger zur Kenntnis genommen und jedenfalls nicht ansatzweise zum Gegenstand ausgedehnter politischer Debatten - so heiß umstritten auch die tatsächlichen außenpolitischen Maßnahmen dieser Jahre waren, vor allem die Kriegseinsätze gegen Serbien, Afghanistan und den Irak. Auch die Kardinalaufgabe der auswärtigen Politik sahen die amerikanischen Demokraten, Labour und Rot-Grün darin, die hinter der Globalisierung verborgenen Kräfte zu steuern. Der deutsche Außenminister Joschka Fischer postulierte schon kurz nach seinem Amtsantritt eine „Außenpolitik, die sich an den Zielen und Herausforderungen des 21. Jahrhunderts in einer globalisierten, in der einen Welt orientiert“ ${ }^{77}$ Die Clinton-Regierung schien, nachdem sie anfänglich noch mühselig nach einem zeitgemäßen Äquivalent zum Containment gesucht hatte, Ende des Jahrzehnts endgültig bei sich selbst angekommen. Nun konnte sie ihr gesamtes auswärtiges Handeln unter das Bestreben subsumieren, die weltweite Globalisierung angemessen zu gestalten. ${ }^{78}$ Der Nationale Sicherheitsberater Samuel Berger resümierte am Ende von Clintons Amtszeit nicht ohne Stolz: „Präsident Clinton erkannte früher als die meisten, dass die durchdringendste Kraft in unserer Welt die Globalisierung ist. " ${ }^{\text {" }}$

Vor diesem Horizont schrieben die Regierungen einem breiten Spektrum transnationaler Probleme ausschlaggebende Bedeutung zu. Diese reichten von Umweltgefahren wie dem Klimawandel und der Energiepolitik über internationale Kriminalität und - befördert durch die islamistischen Anschläge in den USA 2001 und London 2005 - Terrorismus bis hin zu Epidemien wie AIDS, Menschenrechtsfragen und dem Wachstum der Weltbevölkerung. Schließlich erschien es ihnen als dringlich, auf die Verbesserung der Lebensverhältnisse im globalen Süden hinzuwirken. Die grassierende Armut sollte bekämpft und damit die weltweite Ungleichheit verringert werden, wofür es Schulden zu erlassen und die Entwicklungshilfe auszuweiten galt. Dabei richtete sich der Fokus vor allem auf den afrikanischen Kontinent. ${ }^{80}$

In diesen Prioritätensetzungen schlug sich eine prononcierte sicherheitspolitische Konzeption nieder. Denn grenzüberschreitende Probleme und weltweite Schieflagen zu beseitigen, galt den außenpolitischen Experten vor allem deshalb

\footnotetext{
77 Deutscher Bundestag, Stenographischer Bericht, 3. Sitzung, 14. Wahlperiode, 10.11.1998, S. 112; dip21.bundestag.de/dip21/btp/14/14003.pdf [26.3.2020].

${ }^{78}$ Vgl. Clinton, Remarks on United States Foreign Policy, 26.2.1999.

${ }^{79}$ Samuel R. Berger, A Foreign Policy for the Global Age, in: Foreign Affairs 70 (2000), S. 22-39, hier S. 23.

${ }^{80}$ Vgl. Michael Clarke, Foreign Policy, in: Seldon (Hrsg.), Blair's Britain, S. 593-614.
} 
als existenziell, weil sie andernfalls den internationalen Frieden gefährden könnten. Dieser Gedanke beschränkte sich nicht auf den viel diskutierten Zusammenhang von Armut, gescheiterten Staaten und transnationalem Terrorismus. Die Globalisierung, so die umfassendere Situationsanalyse im zentralen Strategiepapier des britischen Foreign Office von 2003, mache es

\begin{abstract}
„Kriminellen leichter, über Grenzen hinweg zu operieren. Grenzübergreifende Probleme wie die Weiterverbreitung von Massenvernichtungswaffen, internationaler Terrorismus und Verbrechen sind eine wachsende Gefahr. Krankheiten, Umweltveränderungen und Ressourcenknappheit können Frieden und Entwicklung unterhöhlen. Interdependenz bedeutet, dass reiche Länder leichter von den Folgen von Unruhen oder Feindseligkeiten in ärmeren Weltgegenden betroffen werden können.“ 81
\end{abstract}

Mit ihrer Themenagenda definierten die amerikanische, die britische und die deutsche Regierung den Gegenstandsbereich der Außenpolitik markant um, wenn man es mit der Politik vergleicht, die die Länder in den 1980er und frühen 1990er Jahren verfolgt hatten. Gänzlich neu war sie allerdings nicht. Denn bereits in den 1970er Jahren waren transnationale Entwicklungen in den Fokus der westlichen außenpolitischen Community gerückt - und es waren nahezu dieselben gewesen, die auch in den späten 1990ern als vorrangig identifiziert wurden. Auch damals hatte man sie als eine Frage der nationalen und internationalen Sicherheit begriffen. Ihre Virulenz war in letzter Instanz ebenfalls auf die Gesamtdynamik einer sich rasant verwebenden Welt zurückgeführt worden; der Begriff, auf den Politiker und Wissenschaftler diesen Zusammenhang gebracht hatten, lautete Interdependenz. ${ }^{82}$ Die außenpolitischen Innovationen der Jahrhundertwende stellten somit, ohne dass dies ausgesprochen und vielleicht auch erkannt worden wäre, in hohem Maße eine Wiederaufnahme der Aufbrüche der 1970er Jahre dar. Dabei prägten sie die Außenpolitik der Bundesrepublik, nicht aber die der beiden anderen Länder, nunmehr deutlich stärker als 20 Jahre zuvor. Zudem blieben die transnationalen Themen jetzt dauerhaft auf dem Tapet der internationalen Diskussion.

Doch reichte die Globalisierungsdiagnose auch im Bereich des auswärtigen Handelns über den Status einer Beobachtungskategorie hinaus, mit der sich drängende Probleme identifizieren ließen. Sie flankierte auch die zum Teil markante Selbstaffirmation, die die drei Regierungen im internationalen Raum an den Tag legten. Das Gewicht der politischen Traditionen sorgte dafür, dass der außenpolitische Umbruch, der sich damit verband, unterschiedlich stark ausgeprägt

\footnotetext{
${ }^{81}$ UK International Priorities. A Strategy for the FCO, London 2003, S. 13. Vgl. ähnlich Berger, Foreign Policy.

${ }^{82}$ Vgl. Jan Eckel, Die Ambivalenz des Guten. Menschenrechte in der internationalen Politik seit den 1940ern, Göttingen 2015, S. 435-582, und Martin Deuerlein, Das Zeitalter der Interdependenz. Globalistisches Denken und internationale Politik 1960-1980, Göttingen 2020 (i. E.).
} 
war. In den USA trat er, jenseits von Außenwirtschaftsfragen, vergleichsweise am schwächsten in Erscheinung. Nachdem mit der Sowjetunion der jahrzehntelange Hauptantagonist weggefallen war, entstand eine Situation der Offenheit, in der durchaus kontrovers diskutiert wurde, ob sich das Land überhaupt noch global engagieren solle. ${ }^{83}$ Diese fluide Phase hielt letztlich an, bis der War on Terror der einzigen verbliebenen Supermacht eine neue Mission verschaffte. Nichtsdestoweniger fand die Clinton-Regierung den wichtigsten Grund dafür, nach wie vor als globale Ordnungsmacht aufzutreten, in der Dynamik zunehmender weltweiter Vernetzung. Ihre ursprüngliche Zielvision, der zufolge es geboten war, Demokratie, Marktwirtschaft und überhaupt die Werte der westlichen Welt über den Globus auszubreiten, weil darin die langfristig wirksamste Politik lag, um weltweiten Frieden und Prosperität zu gewährleisten, verschwand dadurch nicht. Doch wurde das Democratic Enlargement zusehends vom Globalisierungsgedanken überlagert (was sich ähnlich auch in der britischen Regierung erkennen ließ). ${ }^{84}$

In der Praxis folgten die USA dabei keinem konsistenten Kurs. Der humanitäre Interventionismus, den die Demokraten von der republikanischen Vorgängerregierung geerbt hatten, schien nach dem somalischen Fiasko 1992/93 bereits beendet, bevor er richtig begonnen hatte. Aus den Bürgerkriegen im zerfallenden Jugoslawien hielt sich die Clinton-Regierung jahrelang heraus, bevor das Massaker von Srebrenica 1995 den nationalen und internationalen Handlungsdruck stark anwachsen ließ. Die allseits als erfolgreich gewerteten Militärschläge gegen Serbien im Kosovo-Konflikt 1999 waren dann die späte Rehabilitierung einer humanitär motivierten Einmischungspraxis, die es zuvor bestenfalls in Ansätzen gegeben hatte.

Der außenpolitische Sprung, zu dem die rot-grüne Bundesregierung ansetzte, war dagegen sehr viel größer, was wiederum an der ausgeprägten internationalen Zurückhaltung lag, die sich das Land in den Jahrzehnten zuvor auferlegt hatte. Die Regierung Schröder bekundete einen robusten Willen zur außenpolitischen Selbstbehauptung. Sie strebte unumwunden an, einen Platz auf dem internationalen Parkett einzunehmen, der dem „Selbstbewusstsein einer erwachsenen Nation“ angemessen sei. ${ }^{85}$ Diese Absicht materialisierte sich bereits in der deutschen Beteiligung an der Kosovo-Intervention, wenngleich diese auch dazu gedacht war, amerikanische Zweifel an der Bündnisloyalität einer linken deutschen Regierung zu zerstreuen. Auch die deutsche Führungsrolle bei der anschließenden Suche nach einer diplomatischen Lösung auf dem Balkan ging in diese Richtung.

Der Gedanke, die Bundesrepublik als aktiven internationalen Mitspieler zu etablieren, speiste ebenfalls die deutsche Haltung zum amerikanischen „Krieg gegen den Terror“ zwischen 2001 und 2003. Verstand der Bundeskanzler die Beteiligung am Krieg gegen Afghanistan zunächst als „aktive Solidarität“, die „Deutschlands

\footnotetext{
${ }^{83}$ Vgl. Dumbrell, Clinton's Foreign Policy.

${ }^{84}$ Vgl. Anthony Lake, From Containment to Enlargement, 21.9.1993, S. 4 f.; babel.hathitrust. org $/ \mathrm{cgi} / \mathrm{pt}$ ?id=uc1.31210024872861;view=1up;seq=1 [10.2.2020].

${ }^{85}$ So Schröder erstmals in seiner Regierungserklärung vom November 1998: Deutscher Bundestag, Stenographischer Bericht, 3. Sitzung, 14. Wahlperiode, 10.11.1998, S. 61.
} 
Verantwortung in der Welt angemessen" sei, so folgte die offene Konfrontation mit der Regierung George W. Bushs über den Irakkrieg letztlich der gleichen Begründung: Deutschland sei ein „selbstbewusstes Land“, ${ }^{86}$ das für „Abenteuer [...] nicht zur Verfügung “87 stehe. Bei alledem waren es gerade die wahrgenommenen Probleme der globalisierten Welt, in denen die rot-grüne Regierung vielversprechende außenpolitische Profilierungsmöglichkeiten erblickte. So setzte sie sich besonders für das Kyoto-Protokoll, den Internationalen Strafgerichtshof, für Friedensmissionen und den Kampf gegen die weltweite Armut ein. Krönen sollte dieses Engagement ein permanenter deutscher Sitz im Sicherheitsrat der Vereinten Nationen, der Rot-Grün allerdings verwehrt blieb. ${ }^{88}$ Mit alledem unterschied sich die Globalisierungsrede der Bundesregierung auf diesem Feld nicht grundlegend von derjenigen in den Sozialstaatsdebatten. Mit Blick auf den internationalen Raum kamen jedoch die verheißungsvollen Noten des Verflechtungsarguments wesentlich stärker zum Tragen, während in der Begründung der inneren Reformen ein defensivfatalistischer Ton vorherrschte.

Die größte Bedeutung gewann das Globalisierungsdenken allerdings für die britische Außenpolitik. Tony Blair wollte Großbritannien, in engstem Zusammenschluss mit den USA, in eine führende internationale Gestaltungsmacht verwandeln. Wohl stärker als alle seine Vorgänger seit Ende des Zweiten Weltkriegs war er bereit, dafür auch militärische Mittel einzusetzen. ${ }^{89}$ Das deutete sich bereits in den von den Vereinten Nationen nicht autorisierten Angriffen auf den Irak an, die er Ende 1998 an der Seite der USA durchführen ließ. Ihre konzeptionell schlüssige Grundlage erhielt die ausgreifende Zielrichtung der britischen Außenpolitik dann im Zuge des Kosovokriegs. Das humanitäre Interventionsgebot seiner Doctrine of the International Community, die als Keimzelle des Gedankens der Schutzverantwortung gelten kann, entwickelte der Premierminister aus der vermeintlichen Grundtatsache des globalisierten internationalen Raums im fin de siècle. Dass westliche Staaten gegen Serbien intervenierten, sei darauf zurückzuführen, dass „die Welt sich auf eine grundlegendere Weise verändert hat. Die Globalisierung hat unsere Wirtschaften und Arbeitsweisen verändert. Aber die Globalisierung ist nicht nur wirtschaftlich. Sie ist auch ein politisches und ein Sicherheitsphänomen. Wir leben in einer Welt, in der Isolationismus keine Existenzberechtigung mehr hat. ${ }^{90}$ Dies machte Blair an Finanzmärkten und Handel, an der Verbreitung von Atomwaffen und Dro-

\footnotetext{
${ }^{86}$ Stephan Bierling, Vormacht wider Willen. Deutsche Außenpolitik von der Wiedervereinigung bis zur Gegenwart, München 2014, S. 98. Vgl. auch Michael Herkendell, Deutschland: Ziviloder Friedensmacht? Außen- und sicherheitspolitische Orientierung der SPD im Wandel (1982-2007), Bonn 2012.

87 Zit. nach Wolfrum, Rot-Grün, S. 413.

${ }^{88}$ Vgl. Bierling, Vormacht.

${ }^{89}$ Vgl. Clarke, Foreign Policy, in: Seldon (Hrsg.), Blair's Britain, und Matthew Jamison, Humanitarian Interventions since 1990 and „Liberal Interventionism“, in: Brendan Simms/ D. J. B. Trim (Hrsg.), Humanitarian Intervention. A History, Cambridge/New York 2011, S. 365-380.

${ }^{90}$ Tony Blair, Speech in Chicago, 24.4.1999; www.britishpoliticalspeech.org/speech-archive. htm?speech=279 [11.2.2020].
} 
genhandel, an Umweltschäden und der Verschuldung des globalen Südens fest, aber eben auch an gewaltsamen Konflikten und Menschenrechtsverletzungen. Die wechselseitige Abhängigkeit der Weltregionen definierte er als eine sicherheitspolitische und zugleich als eine moralische Frage. Aus beidem ergab sich ein Imperativ des militärischen Eingreifens. Dies ging deutlich über die Positionierung der USA hinaus, die mit Blick auf den Kosovo stärker auf die ethische Verwobenheit im globalen Zeitalter verwies, die es verhindere, fernem Leid gegenüber gleichgültig zu sein: „Was an irgendeinem Ort geschieht, kann überall spürbar sein. “91 Aus diesem Grund könne sich die Betroffenheit der amerikanischen Zivilgesellschaft nunmehr leichter in innenpolitischen Handlungsdruck übersetzen.

Die Konzeption, die Blair 1999 im Zusammenhang dargelegt hatte, blieb für die Labour-Regierung auch anschließend handlungsleitend. Das zeigte sich (nach einer begrenzten Militäroperation in Sierra Leone im Jahr 2000) besonders in der britischen Unterstützung für den amerikanischen Kampf gegen den transnationalen islamistischen Terrorismus. Die Anschläge vom 11. September 2001 wirkten auf Blair wie der bislang stärkste Beleg für die globalistische Weltsicht, die er seit Jahren vertreten hatte. In einer Rede wenige Wochen nach den Angriffen stellte der britische Premier daher mit nochmals gewachsener Überzeugung die Diagnose der verwobenen Welt in den Mittelpunkt seiner Reflexion. Doch diesmal lag die Konsequenz nicht lediglich in einer interventionistischen Agenda, sondern im Entwurf einer umfassenden Weltordnungspolitik. Sie enthielt den groß angelegten Plan, den afrikanischen Kontinent zugleich zu demokratisieren und aus seinen Notlagen zu befreien, dem Klimawandel entgegenzutreten und Frieden im Nahen Osten zu schaffen. ${ }^{92}$ Dass die Folgen, die der von Blair leidenschaftlich unterstützte Irakkrieg für die Region und darüber hinaus hatte, mit dieser globalen Vision scharf zu kontrastieren, ja ihre eklatantesten inhärenten Schwächen aufzuzeigen schien, war dann der wichtigste Grund, warum Tony Blair sein Amt 2007 als einer der unpopulärsten Premierminister der britischen Geschichte niederlegen sollte. ${ }^{93}$

\section{Globalisierung als folgenreiche Selbstbeschreibung}

Der Gedanke einer schleunig zusammenwachsenden Welt entwickelte sich in der demokratischen Regierung der USA, der britischen Labour-Partei und den modernisierungswilligen Teilen von SPD und Grünen seit Mitte der 1990er Jahre zu einer einflussreichen Leitvorstellung. Dies geschah im Kontext einer weitgefächerten gesellschaftlichen Diskussion, in der unterschiedliche Positionen und gegensätzliche Bewertungen aufeinanderprallten, die grundlegenden Zusammenhänge aber von den wenigsten bestritten wurden. Die meisten Beobachterinnen und Beobachter

\footnotetext{
${ }^{91}$ Bill Clinton, Remarks at the „Millennium Around the World“ Celebration, 31.12.1999; www.presidency.ucsb.edu / documents / remarks-the-millennium-around-the-world-celebration [11.2.2020]. Vgl. auch Berger, Foreign Policy.

${ }^{92}$ Vgl. Tony Blair, Leader's Speech, Brighton, 2.10.2001; www.britishpoliticalspeech.org/ speech-archive.htm?speech=186 [11.2.2020].

${ }^{93}$ Vgl. Clarke, Foreign Policy, in: Seldon (Hrsg.), Blair's Britain.
} 
in den drei westlichen Ländern, einschließlich der linken und linksliberalen Regierungen, sahen die Globalisierungsdynamik in zahlreichen Lebensbereichen am Werk. Sie prägte die wichtigen nationalgesellschaftlichen Problemfelder in dieser Sicht ebenso wie die weltpolitischen - Innen- und Außenpolitik ließen sich ohnehin, wie eine weitere ubiquitäre Formel dieser Jahre besagte, gar nicht mehr sauber voneinander trennen, sondern gingen fließend ineinander über. Die Wahrnehmung wachsender weltweiter Verflechtung brachte es zum einen mit sich, dass Handlungsbedarf identifiziert wurde, was sich unter anderem in der Aufmerksamkeit für transnationale Gefahren äußerte. Zum anderen bestärkte sie bestimmte politische Lösungsansätze oder legte sie überhaupt erst nahe, was sich etwa am Plädoyer für die Handelsliberalisierung zeigte. Auf diese Weise kam ihr in der Praxis ein erhebliches Gewicht zu. Das Globalisierungsdenken floss in zwei der wichtigsten neuen Politikansätze der 1990er und frühen 2000er Jahre, die Sozialstaatsreformen und den humanitär begründeten Militärinterventionismus, maßgeblich ein. Dabei speiste es auch die ganze Ambivalenz, die diesen Konzeptionen innewohnte. Es begründete den einschneidenden sozialpolitischen Umbau, der sich freilich auf dem sandigen Boden einer höchst uneindeutigen ökonomischen Analystik vollzog. Ebenso schärfte es das Bewusstsein für die destabilisierenden Wirkungen, die von Konflikten, Repression und Verelendung in zum Teil weit entfernten Weltgegenden ausgehen konnten, lag damit aber auch an der Wurzel einer Einmischungspraxis, die nie ohne problematische Nebenfolgen blieb.

Schon weil sich das Globalisierungsdenken sachlogisch weit über ökonomische Fragen hinaus erstreckte, lässt es sich nicht mit einer neoliberalen Wirtschaftsideologie in eins setzen. Rhetorisch wurde es durchaus verwendet, um Liberalisierungsschritte oder Sozialkürzungen als geboten erscheinen zu lassen. Doch bildeten die wirtschafts- und sozialpolitischen Konzeptionen der drei Regierungen, die auf die Stimulierung des Markts und eine Reduktion sozialstaatlicher Leistungen abhoben, den Teil einer Orthodoxie, die von zahlreichen, weltanschaulich und politisch durchaus nicht homogenen Akteuren geteilt wurde; nicht zuletzt verschrieben sich ihr in der Bundesrepublik alle Parteien bis auf die extreme Linke. Der Globalisierungsgedanke stand in einem bekräftigenden Wechselverhältnis mit diesen wirtschaftspolitischen Rezepten: Er verschaffte ihnen zusätzliche Plausibilität und wurde umgekehrt im Blick auf die weltwirtschaftlichen Zusammenhänge selbst erhärtet.

Ideengeschichtlich betrachtet, lag die Globalisierungsvorstellung sozusagen tiefer, als es die zeitgenössische globalisierungskritische Lesart erfasste. Grundsätzlich war diese Vorstellung ein Ausfluss des Versuchs, gedanklich mit dem Bündel politischer, wirtschaftlicher und sozialer Veränderungen zurechtzukommen, die sich in den Jahren um die Jahrhundertwende ereigneten. Dabei ging es um erfolgversprechende politische Formeln, aber auch um die Suche nach Erklärungen und nach angemessenen Reaktionen. Wenn es im politischen Diskurs einen ideologischen Zug gab, so bestand er darin, dass sich die Deutung der rasanten weltweiten Integration in ein stark verfestigtes weltanschauliches Modell verwandeln konnte, in das sich praktisch alle Beobachtungen einfügen ließen - oder, so konnte es bisweilen scheinen, irgendwie eingefügt werden mussten. Abweichende Er- 
klärungsmöglichkeiten, gegenläufige Tendenzen oder alternative Handlungsoptionen blieben dagegen ausgeblendet. Die Auffassung etwa, dass der Sozialstaat zumindest in Teilbereichen womöglich einen positiven Faktor des volkswirtschaftlichen Standorts bildete, wenn Unternehmen doch offensichtlich nach politischer Berechenbarkeit und einem hohen Qualifikationsniveau suchten, konnte sich über engste akademische Zirkel hinaus nicht behaupten. ${ }^{94}$

Die Suche nach einem adäquaten Verständnis der Gegenwart erzeugte dann wiederum eine politische Wirklichkeitsauffassung, die über einzelne Reform- und Handlungskonzeptionen hinausreichte. Die Globalisierungsrede betonte die Neuartigkeit der Wandlungsprozesse, die sich um die Jahrhundertwende vollzogen, schrieb den Wirkungen grenzüberschreitender Verknüpfungen ein immenses Gewicht zu und versetzte die Politik in einen Modus der Dramatik. In der historischen Analyse dürfte manches davon nicht unerheblich zu relativieren sein. Angst vor islamistisch motivierten Terroranschlägen in den heimischen Großstädten hatten westliche Gesellschaften vor Mitte der 1990er Jahre tatsächlich nicht haben müssen; aber die Frage, ob sich die Regierungen in Fällen der Massengewalt im Ausland einmischen sollten, hatte sich prinzipiell auch vor dem Kosovo-Konflikt schon gestellt. Die Zunahme der Zahl transnationaler Unternehmen und der ausländischen Direktinvestitionen wiederum hatte in den 1970er Jahren begonnen, und dass die Beschleunigung in den 1990er Jahren eine qualitative Veränderung darstelle, wurde selbst von einigen Zeitgenossen bestritten. Jedenfalls waren die Probleme der Wirtschaft und des Arbeitsmarkts, die in der Bundesrepublik diskutiert wurden, der Sache nach seit den 1980er Jahren in hohem Maße gleich geblieben.${ }^{95}$ Es wäre historisch wenig aufschlussreich, wollte man solche modifizierenden Überlegungen dafür verwenden, zeitgenössische Diagnosen als unzutreffend zu entlarven. Vielmehr unterstreichen sie abschließend, dass es sich bei der Globalisierungsvorstellung um eine Form der politischen und gesellschaftlichen Selbstverständigung und der Verarbeitung von Wandel handelte, die womöglich bis heute ihre Prägekraft nicht verloren hat, aber trotzdem ein historisches Produkt der Jahrhundertwende darstellt. Und sie lassen es als ratsam erscheinen, die zeitgenössischen Wahrnehmungen nicht interpretatorisch zu duplizieren, indem man die Globalisierung als Basisprozess jener Jahre voraussetzt oder sie sogar zur Signatur der Epoche erklärt, die sich so sehr an ihr abgearbeitet hat - außer vielleicht in diesem Sinne.

\footnotetext{
${ }^{94}$ Vgl. Colin Hay, Globalization, Economic Change and the Welfare State. The „Vexatious Inquisition of Taxation“, in: Sykes/Palier/Prior (Hrsg.), Globalization, S. 38-58, und Garrett/Mitchell, Globalization.

${ }^{95}$ Vgl. Herbert, Geschichte, S. 1231-1237.
} 